\title{
JACOBI AND LAGUERRE QUASI-ORTHOGONAL APPROXIMATIONS AND RELATED INTERPOLATIONS
}

\author{
GUO BEN-YU, SUN TAO, AND ZHANG CHAO
}

\begin{abstract}
In this paper, we investigate Jacobi quasi-orthogonal approximation and generalized Jacobi-Gauss-Lobatto interpolation. We also propose Laguerre quasi-orthogonal approximation and generalized Laguerre-Gauss-Radau interpolation. A series of sharp results on these approximations are established, which are applicable to spectral and pseudospectral methods for mixed nonhomogeneous boundary value problems of high order.
\end{abstract}

\section{INTRODUCTION}

The Jacobi approximation plays an important role in mathematical analysis and its applications. Especially, the Legendre and Chebyshev approximations have been used widely for spectral and pseudospectral methods of differential equations; see [3, 5, 6, 10, 11, 12, 29] and the references therein. Some authors developed the Jacobi spectral and pseudospectral methods for singular differential equations, and some problems defined on unbounded domains and axisymmetric domains; see, e.g., [2, 13, 14. We mostly considered second order problems. But, it is also important to solve high order problems arising in science and engineering numerically; see [1, 4, 8, 15] and the references therein. Guo, Shen and Wang [18, 19] proposed the generalized Jacobi approximation, which enlarged applications of the spectral method. In actual computation, the pseudospectral method is more preferrable. Its mathematical foundation is the related interpolation. Canuto and Quarteroni first studied systematically the Legendre-Gauss-Lobatto and Chebyshev-Gauss-Lobatto interpolations in the standard Sobolev space; see [6. Bernardi and Maday 3 derived some precise results on the generalized Legendre-Gauss-Lobatto interpolation. Guo and Wang [21, and Guo and Zhang [27] studied the Jacobi-Gauss type interpolations. However, it is not easy to design proper spectral and pseudospectral methods for high order problems with mixed nonhomogeneous boundary conditions.

On the other hand, more and more attention has been paid recently to numerical methods for unbounded domains. For problems defined on the half-line and related problems, we often used the Laguerre spectral and pseudospectral methods; see 17, 23, 26, 28, 30, 31, 32, 33, 36 and the references therein. They are also applicable to certain exterior problems; see, e.g., [7, 20, 24, 25. Whereas, the existing results on the Laguerre approximation are not optimal. Moreover, it is not easy to deal

Received by the editor November 17, 2009 and, in revised form, August 17, 2011.

2010 Mathematics Subject Classification. Primary 41A10, 41A05, 41A30, 65L60.

Key words and phrases. Jacobi quasi-orthogonal approximations, Jacobi-Gauss-Lobatto interpolation, Laguerre quasi-orthogonal approximation, Laguerre -Gauss-Radau interpolation.

The work of this author is supported in part by NSF of China N. 11171227, Fund for Doctor Degree Authority of Chinese Educational Ministry N. 20080270001, Shanghai Leading Academic Discipline Project N. S30405 and Fund for E-institute of Shanghai Universities N. E03004. 
with mixed nonhomogeneous boundary value problems of high order, defined on unbounded domains.

In this work, we investigate the Jacobi and Laguerre quasi-orthogonal approximations and the related interpolations. In the next section, we introduce the Jacobi quasi-orthogonal approximation and establish the basic approximation results. In Section 3, we propose a new generalized Jacobi-Gauss-Lobatto interpolation. In Section 4, we introduce a new family of generalized Laguerre functions which are mutually orthogonal with the weight $x^{\alpha} e^{-\beta x}, \alpha$ being any real number and $\beta>0$. Then, we focus on the special and mostly useful case with integer $\alpha \leq-1$, and derive the optimal approximation result. We also develop the generalized Laguerre quasi-orthogonal approximation. In Section 5, we consider the generalized Laguerre-Gauss-Radau interpolation.

The new approximations proposed in this paper are appropriate for spectral and spectral element methods, and pseudospectral and pseudospectral element methods, for various mixed nonhomogeneous boundary value problems of high order. The approximation results obtained in this work generalize and improve the existing results essentially, and provide new and powerful tools for numerical analysis of high order methods.

\section{JACOBI QUASI-ORTHOGONAL APPROXIMATION}

2.1. Generalized Jacobi functions. Let $\Lambda=\{x|| x \mid<1\}$ and $\chi(x)$ be a certain weight function. For any integer $r \geq 0$, we define the weighted Sobolev space $H_{\chi}^{r}(\Lambda)$ as usual, with the inner product $(u, v)_{r, \chi}$, the semi-norm $|v|_{r, \chi}$ and the norm $\|v\|_{r, \chi}$. In particular, we denote by $(u, v)_{\chi}$ and $\|v\|_{\chi}$ the inner product and the norm of $L_{\chi}^{2}(\Lambda)$, respectively. The space $H_{0, \chi}^{r}(\Lambda)$ stands for the closure in $H_{\chi}^{r}(\Lambda)$ of the set $\mathcal{D}(\Lambda)$ consisting of all infinitely differentiable functions with compact support in $\Lambda$. We omit the subscript $\chi$ in the notation, whenever $\chi(x) \equiv 1$.

In this work, we shall use a specific family of generalized Jacobi polynomials. Let $\chi^{(\sigma, \lambda)}(x)=(1-x)^{\sigma}(1+x)^{\lambda}, \sigma, \lambda>-1$, and let $J_{l}^{(\sigma, \lambda)}(x)$ be the Jacobi polynomials of degree $l$. For any integers $m$ and $n$, we set

$$
Y_{l}^{(m, n)}(x)= \begin{cases}(1-x)^{m}(1+x)^{n} J_{l-m-n}^{(m, n)}(x), & m, n \geq 1, l \geq m+n \\ (1-x)^{m} J_{l-m}^{(m,-n)}(x), & m \geq 1, n<1, l \geq m \\ (1+x)^{n} J_{l-n}^{(-m, n)}(x), & m<1, n \geq 1, l \geq n \\ J_{l}^{(-m,-n)}(x), & m, n<1, l \geq 0 .\end{cases}
$$

According to (2.25) and (2.26) of [19, for integers $m, n, k \geq 1$ and $n \leq m$, we have that

$$
\partial_{x}^{k} Y_{l}^{(m, n)}(x)=E_{k, l}^{(m, n)} Y_{l-k}^{(m-k, n-k)}(x), \quad l \geq \max (m+n, k),
$$

where

$$
E_{k, l}^{(m, n)}=\left\{\begin{array}{l}
(-2)^{k} \prod_{i=1}^{k}(l-m-n+i), \quad k \leq n \leq m, \\
(-1)^{k} 2^{n}\left(\prod_{i=1}^{n}(l-m-n+i)\right)\left(\prod_{i=0}^{k-n-1}(l-n-i)\right), \quad n<k \leq m, \\
(-1)^{m} \frac{\Gamma(l+k-m-n+1)}{2^{k-m-n} \Gamma(l-n+1)}\left(\prod_{i=1}^{n}(l-m-n+i)\right)\left(\prod_{i=0}^{m-n-1}(l-n-i)\right), \\
n<m \leq k .
\end{array}\right.
$$


Meanwhile, for integers $m, n, k \geq 1$ and $m \leq n$, we get

$$
\partial_{x}^{k} Y_{l}^{(m, n)}(x)=(-1)^{\mu} E_{k, l}^{(n, m)} Y_{l-k}^{(m-k, n-k)}(x),
$$

where $\mu=0, k, n$ for the cases $k \leq m \leq n, m<k \leq n$, and $m<n \leq k$, respectively.

The set of all polynomials $Y_{l}^{(m, n)}(x)$ is a complete $L_{\chi^{(-m,-n)}}^{2}(\Lambda)$-orthogonal system, namely (see (2.9) of [19]),

$$
\int_{\Lambda} Y_{l}^{(m, n)}(x) Y_{l^{\prime}}^{(m, n)}(x) \chi^{(-m,-n)}(x) d x=\gamma_{l}^{(m, n)} \delta_{l, l^{\prime}}
$$

where $\delta_{l, l^{\prime}}$ is the Kronecker symbol, and

$$
\gamma_{l}^{(m, n)}= \begin{cases}\frac{2^{m+n+1} \Gamma(l-m+1) \Gamma(l-n+1)}{(2 l-m-n+1) \Gamma(l+1) \Gamma(l-m-n+1)}, & m, n \geq 1, l \geq m+n, \\ \frac{2^{m-n+1} \Gamma(l+1) \Gamma(l-m-n+1)}{(2 l-m-n+1) \Gamma(l-m+1) \Gamma(l-n+1)}, & m \geq 1, n<1, l \geq m, \\ \frac{2^{-m+n+1} \Gamma(l+1) \Gamma(l-m-n+1)}{(2 l-m-n+1) \Gamma(l-m+1) \Gamma(l-n+1)}, & m<1, n \geq 1, l \geq n, \\ \frac{2^{-m-n+1} \Gamma(l-m+1) \Gamma(l-n+1)}{(2 l-m-n+1) \Gamma(l+1) \Gamma(l-m-n+1)}, & m, n<1, l \geq 0 .\end{cases}
$$

Let $l_{m, n}=m+n, m, n, 0$ for the cases $m, n \geq 1, m \geq 1$ and $n<1, m<1$ and $n \geq 1$, and $m, n<1$, respectively. Then, for any $v \in L_{\chi^{(-m,-n)}}^{2}(\Lambda)$,

$$
v(x)=\sum_{l=l_{m, n}}^{\infty} \hat{v}_{l}^{(m, n)} Y_{l}^{(m, n)}(x)
$$

where

$$
\hat{v}_{l}^{(m, n)}=\frac{1}{\gamma_{l}^{(m, n)}} \int_{\Lambda} v(x) Y_{l}^{(m, n)}(x) \chi^{(-m,-n)}(x) d x .
$$

Furthermore, for $m, n \geq 1$, a combination of (2.2)-(2.4) leads to the following equality (cf. the proof of Theorem 2.2 of [19]):

$$
\int_{\Lambda} \partial_{x}^{k} Y_{l}^{(m, n)}(x) \partial_{x}^{k} Y_{l^{\prime}}^{(m, n)}(x) \chi^{(-m+k,-n+k)}(x) d x=\left(E_{k, l}^{(m, n)}\right)^{2} \gamma_{l-k}^{(m-k, n-k)} \delta_{l, l^{\prime}}
$$

Now, for any positive integer $N, \mathcal{P}_{N}(\Lambda)$ stands for the set of all algebraic polynomials of degree at most $N$. Next, for $m, n \geq 1$,

$$
\begin{aligned}
& \mathcal{P}_{N, m, n}^{0}(\Lambda)=\left\{\phi \in \mathcal{P}_{N}(\Lambda) \mid \partial_{x}^{k} \phi(-1)=0 \text { for } 0 \leq k \leq n-1\right. \text { and } \\
& \left.\qquad \partial_{x}^{k} \phi(1)=0 \text { for } 0 \leq k \leq m-1\right\} .
\end{aligned}
$$

We also introduce the finite dimensional set

$$
\mathcal{Q}_{N}^{(m, n)}(\Lambda)=\operatorname{span}\left\{Y_{l}^{(m, n)}(x), l_{m, n} \leq l \leq N\right\} .
$$

Obviously, $\mathcal{Q}_{N}^{(m, n)}(\Lambda)=\mathcal{P}_{N, m, n}^{0}(\Lambda)$ whenever $m, n \geq 1$.

Throughout this paper, we denote by $c$ a generic positive constant independent of any function and $N$. We have an inverse inequality stated below.

Lemma 2.1. For any $\phi \in \mathcal{Q}_{N}^{(m, n)}(\Lambda)$, integers $m, n \geq 1$ and $N \geq \max (m+n, k)$, we get

$$
\left\|\partial_{x}^{k} \phi\right\|_{\chi^{(-m+k,-n+k)}} \leq c N^{k}\|\phi\|_{\chi^{(-m,-n)}} .
$$


Proof. According to (2.4), we have

$$
\|\phi\|_{\chi^{(-m,-n)}}^{2}=\sum_{l=m+n}^{N}\left(\hat{\phi}_{l}^{(m, n)}\right)^{2} \gamma_{l}^{(m, n)} .
$$

Without any loss of generality, we assume $m \geq n$. Then by virtue of (2.7), we get

$$
\left\|\partial_{x}^{k} \phi\right\|_{\chi^{(-m+k,-n+k)}}^{2}=\sum_{l=\max (m+n, k)}^{N}\left(E_{k, l}^{(m, n)}\right)^{2}\left(\hat{\phi}_{l}^{(m, n)}\right)^{2} \gamma_{l-k}^{(m-k, n-k)} .
$$

Clearly, (2.2) and (2.3) imply $E_{k, l}^{(m, n)} \sim l^{k}$ for large $l$. By the Stirling formula,

$$
\Gamma(s+1)=\sqrt{2 \pi s} s^{s} e^{-s}\left(1+O\left(s^{-\frac{1}{5}}\right)\right), \quad s \gg 1 .
$$

Thus, a direct calculation shows that $\gamma_{l}^{(m, n)} \sim l^{-1}$. Thereby, we use (2.8) and (2.9) to obtain

$$
\begin{aligned}
\left\|\partial_{x}^{k} \phi\right\|_{\chi^{(-m+k,-n+k)}}^{2} & \leq \max _{\max (m+n, k) \leq l \leq N} \frac{\gamma_{l-k}^{(m-k, n-k)}\left(E_{k, l}^{(m, n)}\right)^{2}}{\gamma_{l}^{(m, n)}} \sum_{l=m+n}^{N}\left(\hat{\phi}_{l}^{(m, n)}\right)^{2} \gamma_{l}^{(m, n)} \\
& \leq c N^{2 k}\|\phi\|_{\chi^{(-m,-n)}}^{2} .
\end{aligned}
$$

This ends the proof.

2.2. Generalized Jacobi orthogonal approximation. Next, we recall the generalized Jacobi orthogonal approximation. The projection $P_{N, m, n}: L_{\chi^{(-m,-n)}}^{2}(\Lambda) \rightarrow$ $\mathcal{Q}_{N}^{(m, n)}(\Lambda)$ is defined by

$$
\left(P_{N, m, n} v-v, \phi\right)_{\chi^{(-m,-n)}}=0, \quad \forall \phi \in \mathcal{Q}_{N}^{(m, n)}(\Lambda) .
$$

As a special case of (2.39) of [19] (also see (1.8) of [18]), we have the following result.

Lemma 2.2. If $v \in L_{\chi^{(-m,-n)}}^{2}(\Lambda), \partial_{x}^{r} v \in L_{\chi^{(-m+r,-n+r)}}^{2}(\Lambda)$, integers $m, n, r \geq$ $1, N \geq m+n$ and $0 \leq k \leq r \leq N+1$, then

$$
\left\|\partial_{x}^{k}\left(v-P_{N, m, n} v\right)\right\|_{\chi^{(-m+k,-n+k)}} \leq c N^{k-r}\left\|\partial_{x}^{r} v\right\|_{\chi^{(-m+r,-n+r)}} .
$$

For numerical solutions of high order differential equations, we need other orthogonal projections. For this purpose, we introduce the space

$$
H_{m, n, A}^{r}(\Lambda)=\left\{v \mid v \text { is measurable on } \Lambda \text { and }\|v\|_{H_{m, n, A}^{r}}<\infty\right\},
$$

equipped with the following semi-norm and norm,

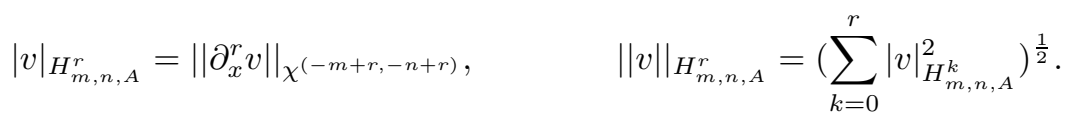

If $v \in H_{m, n, A}^{r}(\Lambda), m, n \geq 1$ and $r \geq \max (m, n)$, then there exist finite traces $\partial_{x}^{k} v(-1)$ for $0 \leq k \leq n-1$, and $\partial_{x}^{k} v(1)$ for $0 \leq k \leq m-1$. Accordingly, we define the space

$$
\begin{aligned}
& H_{0, m, n, A}^{r}(\Lambda)=\left\{v \in H_{m, n, A}^{r}(\Lambda) \mid \partial_{x}^{k} v(-1)=0 \text { for } 0 \leq k \leq n-1,\right. \text { and } \\
& \left.\qquad \partial_{x}^{k} v(1)=0 \text { for } 0 \leq k \leq m-1\right\} .
\end{aligned}
$$


For integers $\max (m, n) \leq \mu \leq m+n$, the projection $P_{N, m, n}^{\mu, 0}: H_{0, m, n, A}^{\mu}(\Lambda) \rightarrow$ $\mathcal{Q}_{N}^{(m, n)}(\Lambda)$ is defined by

$$
\left(\partial_{x}^{\mu}\left(v-P_{N, m, n}^{\mu, 0} v\right), \partial_{x}^{\mu} \phi\right)_{\chi^{(-m+\mu,-n+\mu)}}=0, \quad \forall \phi \in \mathcal{Q}_{N}^{(m, n)}(\Lambda) .
$$

There is a close relation between $P_{N, m, n}^{\mu, 0} v$ and $P_{N, m, n} v$. To show this, let $v \in$ $H_{0, m, n, A}^{\mu}(\Lambda)$ and $\hat{v}_{l}^{(m, n)}$ be the same as in (2.9). Meanwhile,

$$
P_{N, m, n}^{\mu, 0} v(x)=\sum_{l=m+n}^{N} a_{l}^{(m, n)} Y_{l}^{(m, n)}(x) .
$$

Without any loss of generality, we assume $1 \leq n \leq m$. With the aid of (2.2), a direct calculation shows

$$
\begin{aligned}
\partial_{x}^{\mu}\left(v(x)-P_{N, m, n}^{\mu, 0} v(x)\right)= & \sum_{l=\max (m+n, \mu)}^{N} E_{\mu, l}^{(m, n)}\left(\hat{v}_{l}^{(m, n)}-a_{l}^{(m, n)}\right) Y_{l-\mu}^{(m-\mu, n-\mu)}(x) \\
& +\sum_{l=N+1}^{\infty} E_{\mu, l}^{(m, n)} \hat{v}_{l}^{(m, n)} Y_{l-\mu}^{(m-\mu, n-\mu)}(x) .
\end{aligned}
$$

We now put $\phi(x)=Y_{l^{\prime}}^{(m, n)}(x), m+n \leq l^{\prime} \leq N$, in (2.11). Then, we use (2.2), (2.4) and (2.12) to obtain

$$
\left(E_{\mu, l}^{(m, n)}\right)^{2} \gamma_{l-\mu}^{(m-\mu, n-\mu)}\left(\hat{v}_{l}^{(m, n)}-a_{l}^{(m, n)}\right)^{2}=0, \quad m+n \leq l \leq N .
$$

This means

$$
P_{N, m, n}^{\mu, 0} v=P_{N, m, n} v, \quad \forall v \in H_{0, m, n, A}^{\mu}(\Lambda) .
$$

The above matter with Lemma 2.2 leads to the following conclusion.

Lemma 2.3. If $v \in H_{0, m, n, A}^{\mu}(\Lambda), \partial_{x}^{r} v \in L_{\chi^{(-m+r,-n+r)}}^{2}(\Lambda)$, integers $m, n, r \geq 1, N \geq$ $m+n, 0 \leq k \leq r \leq N+1$ and $\max (m, n, k) \leq \mu \leq m+n$, then

$$
\left\|\partial_{x}^{k}\left(v-P_{N, m, n}^{\mu, 0} v\right)\right\|_{\chi^{(-m+k,-n+k)}} \leq c N^{k-r}\left\|\partial_{x}^{r} v\right\|_{\chi^{(-m+r,-n+r)}} .
$$

The above result is applicable to numerical solutions of high order problems with homogeneous Dirichlet boundary condition. The specific case with $\mu=m=n>1$ was considered in 18 .

2.3. Jacobi quasi-orthogonal approximation. For nonhomogeneous Dirichlet, Neumann and mixed boundary conditions, as well as for spectral element methods of high order equations in which the numerical solutions and some of their derivatives should match properly on all common boundaries of adjacent subdomains, we need certain quasi-orthogonal approximations.

We introduce the following polynomials of degree $m+n-1$,

$$
\begin{aligned}
& q_{m, n, j}^{-}(x)=\frac{1}{2^{m} j !}(1-x)^{m} \sum_{l=0}^{n-1-j} \frac{(m+l-1) !}{2^{l} l !(m-1) !}(1+x)^{l+j}, \\
& q_{m, n, j}^{+}(x)=\frac{(-1)^{j}}{2^{n} j !}(1+x)^{n} \sum_{l=0}^{m-1-j} \frac{(n+l-1) !}{2^{l} l !(n-1) !}(1-x)^{l+j} .
\end{aligned}
$$


It can be checked that (cf. Theorem 3.1 of [35])

$$
\begin{array}{lll}
\partial_{x}^{k} q_{m, n, j}^{-}(-1)=\delta_{k, j}, & \partial_{x}^{l} q_{m, n, j}^{-}(1)=0, & 0 \leq j, k \leq n-1,0 \leq l \leq m-1, \\
\partial_{x}^{l} q_{m, n, j}^{+}(-1)=0, & \partial_{x}^{k} q_{m, n, j}^{+}(1)=\delta_{k, j}, & 0 \leq j, k \leq m-1,0 \leq l \leq n-1 .
\end{array}
$$

Now, for any $v \in H_{m, n, A}^{r}(\Lambda)$ and $r \geq \max (m, n)$, we set

$$
v_{m, n, b}(x)=\sum_{j=0}^{n-1} \partial_{x}^{j} v(-1) q_{m, n, j}^{-}(x)+\sum_{j=0}^{m-1} \partial_{x}^{j} v(1) q_{m, n, j}^{+}(x) .
$$

Furthermore, let $\widetilde{v}(x)=v(x)-v_{m, n, b}(x)$. Evidently, $\widetilde{v} \in H_{0, m, n, A}^{r}(\Lambda)$. Therefore, there exists the orthogonal projection $P_{N, m, n}^{\mu, 0} \widetilde{v} \in \mathcal{Q}_{N}^{(m, n)}(\Lambda)$. Following the same idea of [23, 25], we define the Jacobi quasi-orthogonal projection as

$$
P_{N, m, n}^{\mu} v(x)=P_{N, m, n}^{\mu, 0} \widetilde{v}(x)+v_{m, n, b}(x) \in \mathcal{P}_{N}(\Lambda) .
$$

By (2.17), (2.18), and the definition of $P_{N, m, n}^{\mu, 0}$, we derive that

$$
\begin{array}{ll}
\partial_{x}^{k} P_{N, m, n}^{\mu} v(-1)=\partial_{x}^{k} v(-1), & \text { for } 0 \leq k \leq n-1, \\
\partial_{x}^{k} P_{N, m, n}^{\mu} v(1)=\partial_{x}^{k} v(1), & \text { for } 0 \leq k \leq m-1 .
\end{array}
$$

We now turn to the error estimate of the above projection. Obviously,

$$
P_{N, m, n}^{\mu} v(x)-v(x)=P_{N, m, n}^{\mu, 0} \widetilde{v}(x)-\widetilde{v}(x) .
$$

Thus, we use Lemma 2.3 to obtain the following result.

Theorem 2.1. If $v \in H_{m, n, A}^{\max (m, n)}(\Lambda), \partial_{x}^{r} v \in L_{\chi^{(-m+r,-n+r)}}^{2}(\Lambda)$, integers $m, n, r \geq$ $1, N \geq m+n, 0 \leq k \leq r \leq N+1$ and $\max (m, n, k) \leq \mu \leq m+n$, then

$$
\begin{aligned}
& \left\|\partial_{x}^{k}\left(P_{N, m, n}^{\mu} v-v\right)\right\|_{\chi^{(-m+k,-n+k)}}=\left\|\partial_{x}^{k}\left(P_{N, m, n}^{\mu, 0} \widetilde{v}-\widetilde{v}\right)\right\|_{\left.\chi^{(-m+k,-n+k}\right)} \\
& \quad \leq c N^{k-r}\left(\left\|\partial_{x}^{r} v\right\|_{\chi^{(-m+r,-n+r)}}+\left\|\partial_{x}^{r} v_{m, n, b}\right\|_{\left.\chi^{(-m+r,-n+r)}\right) .}\right.
\end{aligned}
$$

Clearly, $\partial_{x}^{r} v_{m, n, b}(x)=0$ for $r \geq m+n$. Therefore, the inequality (2.20) implies

$$
\begin{aligned}
& \left\|\partial_{x}^{k}\left(P_{N, m, n}^{\mu} v-v\right)\right\|_{\chi^{(-m+k,-n+k)}} \\
& \quad \leq c N^{k-r}\left\|\partial_{x}^{r} v\right\|_{\chi^{(-m+r,-n+r)}}, \quad \text { for } r \geq m+n .
\end{aligned}
$$

Next, we consider the case with $\max (m, n) \leq r \leq m+n-1$. Thanks to trace theorem, we get

$$
\begin{aligned}
\left\|\partial_{x}^{r} v_{m, n, b}\right\|_{\chi^{(-m+r,-n+r)}} & \leq c\left(\sum_{j=0}^{n-1}\left|\partial_{x}^{j} v(-1)\right|\right. \\
& \left.+\sum_{j=0}^{m-1}\left|\partial_{x}^{j} v(1)\right|\right) \leq c\|v\|_{H^{\max (m, n)}(\Lambda)} .
\end{aligned}
$$

This, along with $(2.20)$, leads to

$$
\begin{aligned}
& \left\|\partial_{x}^{k}\left(P_{N, m, n}^{\mu} v-v\right)\right\|_{\chi^{(-m+k,-n+k)}} \\
& \quad \leq c N^{k-r}\left(\left\|\partial_{x}^{r} v\right\|_{\chi^{(-m+r,-n+r)}}+\|v\|_{H^{\max (m, n)}(\Lambda)}\right) .
\end{aligned}
$$

It is noted that in many practical cases, the error estimate (2.21) is also valid for $r \leq m+n-1$. Indeed, it has been verified that (see the Appendix of this paper)

$$
\begin{aligned}
& \left\|\partial_{x}^{k}\left(P_{N, m, n}^{\mu} v-v\right)\right\|_{\chi^{(-m+k,-n+k)}} \leq c N^{k-r}\left\|\partial_{x}^{r} v\right\|_{\chi^{(-m+r,-n+r)}}, \\
& \quad \text { for } 1 \leq m, n \leq 4, \max (m, n) \leq r \leq m+n-1 .
\end{aligned}
$$


Remark 2.1. The special cases with $\mu=m=n=1,2$ with their applications to second and fourth order problems, were considered in [16, 24, 25, 27]. Bernardi and Maday considered the same projection with $\mu=m=n$, denoted by $\widetilde{\pi}_{N}^{m}$. By (6.22) of [3], $\left\|v-\tilde{\pi}_{N}^{m} v\right\|_{H^{m}(\Lambda)} \leq c N^{m-r}\|v\|_{H^{r}(\Lambda)}$. Clearly, (2.21), (2.23), and (2.24) give more general and better results.

Remark 2.2. The projection $P_{N, \sigma, \lambda}^{\mu} v$ possesses an interesting property, playing an important role in Petrov-Galerkin spectral method of high and odd order problems. In fact, for any $\phi \in \mathcal{Q}_{N-\sigma+\lambda}^{(\lambda, \lambda)}(\Lambda)$, we have $\partial_{x}^{\lambda} \phi \in \mathcal{P}_{N-\sigma}(\Lambda)$. Accordingly, $q_{\phi}:=$ $\chi^{(\sigma, \lambda)} \partial_{x}^{\sigma+\lambda} \phi \in \mathcal{Q}_{N}^{(\sigma, \lambda)}(\Lambda)$. For any $v \in H_{\sigma, \lambda, A}^{\max (\sigma, \lambda)}(\Lambda)$, let $\widetilde{v}(x)=v(x)-v_{\sigma, \lambda, b}(x) \in$ $H_{0, \sigma, \lambda, A}^{\max (\sigma, \lambda)}(\Lambda)$. Then, we use (2.18), an integration by parts, (2.13) and the definition of $P_{N, \sigma, \lambda} \widetilde{v}$ successfully, to derive that for any $\phi \in \mathcal{Q}_{N-\sigma+\lambda}^{(\lambda, \lambda)}(\Lambda)$ and $\max (\sigma, \lambda) \leq \mu \leq$ $m+n$, we get

$$
\begin{aligned}
& \left(\partial_{x}^{\sigma}\left(P_{N, \sigma, \lambda}^{\mu} v-v\right), \partial_{x}^{\lambda} \phi\right)=\left(\partial_{x}^{\sigma}\left(P_{N, \sigma, \lambda}^{\mu, 0} \widetilde{v}-\widetilde{v}\right), \partial_{x}^{\lambda} \phi\right) \\
& \quad=(-1)^{\sigma}\left(P_{N, \sigma, \lambda}^{\mu, 0} \widetilde{v}-\widetilde{v}, q_{\phi}\right)_{\chi}^{(-\sigma,-\lambda)}=(-1)^{\sigma}\left(P_{N, \sigma, \lambda} \widetilde{v}-\widetilde{v}, q_{\phi}\right)_{\chi^{(-\sigma,-\lambda)}}=0 .
\end{aligned}
$$

\section{Generalized Jacobi-Gauss-Lobatto interpolation}

In this section, we investigate the generalized Jacobi-Gauss-Lobatto interpolation.

Denote by $\zeta_{G, N, j}^{(\sigma, \lambda)}$ the $N+1$ distinct zeros of polynomials $J_{N+1}^{(\sigma, \lambda)}(x)$, arranged in decreasing order. As was shown in 34 , there exist the corresponding Christoffel numbers $\omega_{G, N, j}^{(\sigma, \lambda)}, 0 \leq j \leq N$, such that

$$
\int_{\Lambda} \phi(x) \chi^{(\sigma, \lambda)}(x) d x=\sum_{j=0}^{N} \phi\left(\zeta_{G, N, j}^{(\sigma, \lambda)}\right) \omega_{G, N, j}^{(\sigma, \lambda)}, \quad \forall \phi \in \mathcal{P}_{2 N+1}(\Lambda) .
$$

By (15.3.10) of [34], we have

$$
\omega_{G, N, j}^{(\sigma, \lambda)} \sim \frac{2^{\sigma+\lambda+1} \pi}{N+1}\left(1-\zeta_{G, N, j}^{(\sigma, \lambda)}\right)^{\sigma+\frac{1}{2}}\left(1+\zeta_{G, N, j}^{(\sigma, \lambda)}\right)^{\lambda+\frac{1}{2}} .
$$

We define the nodes and weights of the generalized Jacobi-Gauss-Lobatto interpolation:

$$
\begin{aligned}
\zeta_{N, j}^{(m, n)} & =\zeta_{G, N-m-n, j}^{(m, n)}, \\
\omega_{N, j}^{(m, n)} & =\omega_{G, N-m-n, j}^{(m, n)}\left(1-\zeta_{G, N-m-n, j}^{(m, n)}\right)^{-2 m}\left(1+\zeta_{G, N-m-n, j}^{(m, n)}\right)^{-2 n} \\
& m, n \geq 0,0 \leq j \leq N-m-n .
\end{aligned}
$$

Thanks to (3.2) and (3.3), we have

$$
\omega_{N, j}^{(m, n)} \sim \frac{2^{m+n+1} \pi}{N-m-n+1}\left(1-\zeta_{N, j}^{(m, n)}\right)^{-m+\frac{1}{2}}\left(1+\zeta_{N, j}^{(m, n)}\right)^{-n+\frac{1}{2}} .
$$

Next, we introduce the discrete inner product and norm as follows:

$$
(u, v)_{N, m, n}=\sum_{j=0}^{N-m-n} u\left(\zeta_{N, j}^{(m, n)}\right) v\left(\zeta_{N, j}^{(m, n)}\right) \omega_{N, j}^{(m, n)}, \quad\|v\|_{N, m, n}=(v, v)_{N, m, n}^{\frac{1}{2}} .
$$

For any $\phi \in \mathcal{Q}_{N}^{(m, n)}(\Lambda)$ and $\psi \in \mathcal{Q}_{N+1}^{(m, n)}(\Lambda)$, there are $q_{\phi} \in \mathcal{P}_{N-m-n}(\Lambda)$ and $q_{\psi} \in \mathcal{P}_{N-m-n+1}(\Lambda)$ such that $\phi(x)=\chi^{(m, n)}(x) q_{\phi}(x)$ and $\psi(x)=\chi^{(m, n)}(x) q_{\psi}(x)$. 
Clearly, $q_{\phi} q_{\psi} \in \mathcal{P}_{2 N-2 m-2 n+1}(\Lambda)$. Therefore, we use (3.1) to verify that

$$
\begin{aligned}
& (\phi, \psi)_{\chi^{(-m,-n)}}=\left(q_{\phi}, q_{\psi}\right)_{\chi^{(m, n)}} \\
& =\sum_{j=0}^{N-m-n} q_{\phi}\left(\zeta_{G, N-m-n, j}^{(m, n)}\right) q_{\psi}\left(\zeta_{G, N-m-n, j}^{(m, n)}\right) \omega_{G, N-m-n, j}^{(m, n)} \\
& =\sum_{j=0}^{N-m-n} \phi\left(\zeta_{N, j}^{(m, n)}\right) \psi\left(\zeta_{N, j}^{(m, n)}\right) \omega_{N, j}^{(m, n)}=(\phi, \psi)_{N, m, n}, \\
& \forall \phi \in \mathcal{Q}_{N}^{(m, n)}(\Lambda), \psi \in \mathcal{Q}_{N+1}^{(m, n)}(\Lambda) .
\end{aligned}
$$

For any integer $r \geq 0$, we denote by $C^{r}(\bar{\Lambda})$ the space consisting of all $r$-times differentiable functions. Further, for integer $r \geq \max (m-1, n-1)$,

$$
\begin{aligned}
& C_{0, m, n}^{r}(\bar{\Lambda})=\left\{v \in C^{r}(\bar{\Lambda}) \mid \partial_{x}^{k} v(-1)=0 \text { for } 0 \leq k \leq n-1,\right. \\
& \left.\qquad \partial_{x}^{k} v(1)=0 \text { for } 0 \leq k \leq m-1\right\} .
\end{aligned}
$$

For any $v \in C_{0, m, n}^{\max (m-1, n-1)}(\bar{\Lambda})$ and $m, n \geq 1$, the auxiliary interpolation $\mathcal{I}_{N, m, n} v$ $\in \mathcal{Q}_{N}^{(m, n)}(\Lambda)$ is determined by

$$
\mathcal{I}_{N, m, n} v\left(\zeta_{N, j}^{(m, n)}\right)=v\left(\zeta_{N, j}^{(m, n)}\right), \quad 0 \leq j \leq N-m-n .
$$

Lemma 3.1 (Stability of interpolation). For any $v \in C_{0, m, n}^{\max (m-1, n-1)}(\bar{\Lambda}) \cap$ $H_{m, n, A}^{1}(\Lambda)$, integers $m, n \geq 1$ and $N \geq m+n$, we get

$$
\left\|\mathcal{I}_{N, m, n} v\right\|_{\chi^{(-m,-n)}} \leq c\left(\|v\|_{\chi^{(-m,-n)}}+N^{-1}\left\|\partial_{x} v\right\|_{\chi^{(-m+1,-n+1)}}\right) .
$$

Proof. By virtue of (3.4) and (3.5), we deduce that

$$
\begin{aligned}
& \left\|\mathcal{I}_{N, m, n} v\right\|_{\chi^{(-m,-n)}}^{2}=\sum_{j=0}^{N-m-n} v^{2}\left(\zeta_{N, j}^{(m, n)}\right) \omega_{N, j}^{(m, n)} \\
& \quad \leq \frac{2^{m+n+1} \pi}{N-m-n+1} \sum_{j=0}^{N-m-n} v^{2}\left(\zeta_{N, j}^{(m, n)}\right)\left(1-\zeta_{N, j}^{(m, n)}\right)^{-m+\frac{1}{2}}\left(1+\zeta_{N, j}^{(m, n)}\right)^{-n+\frac{1}{2}} .
\end{aligned}
$$

Let $x=\cos \theta, \theta \in[0, \pi]$ and $\widehat{v}(\theta)=v(\cos \theta)$. By (3.3) and Theorem 8.9.1 of [34],

$$
\theta_{N, j}^{(m, n)}=\theta_{G, N-m-n, j}^{(m, n)}=\frac{1}{N-m-n+1}(j \pi+\mathcal{O}(1)), \quad 0 \leq j \leq N-m-n
$$

where $\mathcal{O}(1)$ is bounded uniformly for all $0 \leq j \leq N-m-n$. We set $a_{0}=$ $\frac{1}{N-m-n+1} \mathcal{O}(1)$ and $a_{1}=\frac{1}{N-m-n+1}((N-m-n) \pi+\mathcal{O}(1))$. Then $\theta_{N, j}^{(m, n)} \in \Delta_{j} \subset$ $\left[a_{0}, a_{1}\right], \Delta_{j}$ being subintervals with the length $\left|\Delta_{j}\right|=\mathcal{O}\left(\frac{1}{N-m-n+1}\right)$. Therefore, by (3.7) and (3.8),

$$
\begin{aligned}
\left\|\mathcal{I}_{N, m, n} v\right\|_{\chi(-m,-n)}^{2} & \leq \frac{c}{N} \sum_{j=0}^{N-m-n} v^{2}\left(\theta_{N, j}^{(m, n)}\right)\left(\sin \frac{\theta_{N, j}^{(m, n)}}{2}\right)^{-2 m+1}\left(\cos \frac{\theta_{N, j}^{(m, n)}}{2}\right)^{-2 n+1} \\
& \leq \frac{c}{N} \sum_{j=0}^{N-m-n} \sup _{\theta \in \Delta_{j}}\left|\widehat{v}(\theta)\left(\sin \frac{\theta}{2}\right)^{-m+\frac{1}{2}}\left(\cos \frac{\theta}{2}\right)^{-n+\frac{1}{2}}\right|^{2} .
\end{aligned}
$$

On the other hand, for any $f \in H^{1}(a, b)$ (see page 32 of [21]),

$$
\max _{a \leq x \leq b}|f(x)| \leq \frac{1}{\sqrt{b-a}}\|f\|_{L^{2}(a, b)}+\sqrt{b-a}\left\|\partial_{x} f\right\|_{L^{2}(a, b)} .
$$


Therefore,

$$
\begin{array}{rl}
\left\|\mathcal{I}_{N, m, n} v\right\|_{\chi^{(-m,-n)}}^{2} & c \frac{c}{N} \sum_{j=0}^{N-m-n}\left(\frac{1}{\left|\Delta_{j}\right|}\left\|\widehat{v}(\theta)\left(\sin \frac{\theta}{2}\right)^{-m+\frac{1}{2}}\left(\cos \frac{\theta}{2}\right)^{-n+\frac{1}{2}}\right\|_{L^{2}\left(\Delta_{j}\right)}^{2}\right. \\
& +\left|\Delta_{j}\left\|\mid \partial_{\theta}\left(\widehat{v}(\theta)\left(\sin \frac{\theta}{2}\right)^{-m+\frac{1}{2}}\left(\cos \frac{\theta}{2}\right)^{-n+\frac{1}{2}}\right)\right\|_{L^{2}\left(\Delta_{j}\right)}^{2}\right) \\
\leq & c\left(\left\|\widehat{v}(\theta)\left(\sin \frac{\theta}{2}\right)^{-m+\frac{1}{2}}\left(\cos \frac{\theta}{2}\right)^{-n+\frac{1}{2}}\right\|_{L^{2}(0, \pi)}^{2}\right. \\
& +N^{-2}\left\|\partial_{\theta} \widehat{v}(\theta)\left(\sin \frac{\theta}{2}\right)^{-m+\frac{1}{2}}\left(\cos \frac{\theta}{2}\right)^{-n+\frac{1}{2}}\right\|_{L^{2}\left(a_{0}, a_{1}\right)}^{2} \\
& \left.+N^{-2}\left\|\widehat{v}(\theta)\left(\sin \frac{\theta}{2}\right)^{-m-\frac{1}{2}}\left(\cos \frac{\theta}{2}\right)^{-n-\frac{1}{2}}\right\|_{L^{2}\left(a_{0}, a_{1}\right)}^{2}\right) \\
\leq & c\left(\left\|\widehat{v}(\theta)\left(\sin \frac{\theta}{2}\right)^{-m+\frac{1}{2}}\left(\cos \frac{\theta}{2}\right)^{-n+\frac{1}{2}}\right\|_{L^{2}(0, \pi)}^{2}\right. \\
& +N^{-2}\left\|\partial_{\theta} \widehat{v}(\theta)\left(\sin _{\frac{1}{2}}\right)^{-m+\frac{1}{2}}\left(\cos \frac{\theta}{2}\right)^{-n+\frac{1}{2}}\right\|_{L^{2}(0, \pi)}^{2} \\
& \left.+N^{-2} \max _{a_{0} \leq x \leq a_{1}} \frac{1}{\sin ^{2} \theta}\left(\| \widehat{v}(\theta)\left(\sin \frac{\theta}{2}\right)^{-m+\frac{1}{2}}\left(\cos \frac{\theta}{2}\right)^{-n+\frac{1}{2}}\right) \|_{L^{2}\left(a_{0}, a_{1}\right)}^{2}\right) \\
\leq & c\left(\|v\|_{\chi^{(-m,-n)}}^{2}+N^{-2}\left\|\partial_{x} v\right\|_{\chi(-m+1,-n+1)}^{2}\right) .
\end{array}
$$

The proof is completed.

We are now in position to estimate the error of interpolation $\mathcal{I}_{N, m, n} v$.

Lemma 3.2. If $v \in H_{0, m, n, A}^{\max (m, n)}(\Lambda), \partial_{x}^{r} v \in L_{\chi^{(-m+r,-n+r)}}^{2}(\Lambda)$, integers $m, n, r \geq 1$, $N \geq m+n$ and $0 \leq k \leq r \leq N+1$, then

$$
\left\|\partial_{x}^{k}\left(\mathcal{I}_{N, m, n} v-v\right)\right\|_{\chi^{(-m+k,-n+k)}} \leq c N^{k-r}\left\|\partial_{x}^{r} v\right\|_{\chi^{(-m+r,-n+r)}} .
$$

Proof. $\mathcal{I}_{N, m, n} v$ is meaningful. We use Lemmas 2.1 and 3.1 to deduce that

$$
\begin{aligned}
& \left\|\partial_{x}^{k}\left(\mathcal{I}_{N, m, n} v-P_{N, m, n} v\right)\right\|_{\chi^{(-m+k,-n+k)}} \\
& \leq c N^{k}\left\|\mathcal{I}_{N, m, n} v-P_{N, m, n} v\right\|_{\chi^{(-m,-n)}}=c N^{k}\left\|\mathcal{I}_{N, m, n}\left(P_{N, m, n} v-v\right)\right\|_{\chi^{(-m,-n)}} \\
& \leq c N^{k}\left(\left\|P_{N, m, n} v-v\right\|_{\chi^{(-m,-n)}}+N^{-1}\left\|\partial_{x}\left(P_{N, m, n} v-v\right)\right\|_{\chi^{(-m+1,-n+1)}}\right) .
\end{aligned}
$$

Consequently, using Lemma 2.2 yields

$$
\begin{aligned}
& \left\|\partial_{x}^{k}\left(\mathcal{I}_{N, m, n} v-v\right)\right\|_{\chi^{(-m+k,-n+k)}} \leq\left\|\partial_{x}^{k}\left(P_{N, m, n} v-v\right)\right\|_{\chi^{(-m+k,-n+k)}} \\
& \quad+\left\|\partial_{x}^{k}\left(\mathcal{I}_{N, m, n} v-P_{N, m, n} v\right)\right\|_{\chi^{(-m+k,-n+k)}} \leq c N^{k-r}\left\|\partial_{x}^{r} v\right\|_{\chi^{(-m+r,-n+r)}} .
\end{aligned}
$$

This ends the proof.

By virtue of (3.5) and Lemma 3.2 with $k=0$, we verify that for any $v \in$ $C_{0, m, n}^{\max (m-1, n-1)}(\bar{\Lambda})$ and $\phi \in \mathcal{Q}_{N}^{(m, n)}(\Lambda)$,

$$
\begin{aligned}
\left|(v, \phi)_{N, m, n}-(v, \phi)_{\chi^{(-m,-n)}}\right| & =\left|\left(\mathcal{I}_{N, m, n} v-v, \phi\right)_{\chi^{(-m,-n)}}\right| \\
& \leq c N^{-r}|| \partial_{x}^{r} v||_{\chi^{(-m+r,-n+r)}}\|\phi\|_{\chi^{(-m,-n)}} .
\end{aligned}
$$

We now turn to the generalized Jacobi-Gauss-Lobatto interpolation for function with nonhomogeneous boundary values. Let $v_{m, n, b}(x)$ be the same as in (2.17). For any $v \in C_{m, n}^{\max (m-1, n-1)}(\bar{\Lambda})$, we set $\widetilde{v}(x)=v(x)-v_{m, n, b}(x)$. Evidently, $\widetilde{v} \in$ $C_{0, m, n}^{\max (m-1, n-1)}(\bar{\Lambda})$. Thus, there exists the interpolation $\mathcal{I}_{N, m, n} \widetilde{v} \in \mathcal{Q}_{N}^{(m, n)}(\Lambda)$. Then, 
following the same idea of [27, we define the new generalized Jacobi-Gauss-Lobatto interpolation as

$$
\mathcal{I}_{L, N, m, n} v(x)=\mathcal{I}_{N, m, n} \widetilde{v}(x)+v_{m, n, b}(x) .
$$

With the aid of (2.16), we assert that

$$
\begin{aligned}
& \mathcal{I}_{L, N, m, n} v\left(\zeta_{N, j}^{(m, n)}\right)=v\left(\zeta_{N, j}^{(m, n)}\right), \quad \text { for } 0 \leq j \leq N-m-n, \\
& \partial_{x}^{k} \mathcal{I}_{L, N, m, n} v(-1)=\partial_{x}^{k} v(-1), \quad \text { for } 0 \leq k \leq n-1, \\
& \partial_{x}^{k} \mathcal{I}_{L, N, m, n} v(1)=\partial_{x}^{k} v(1), \quad \text { for } 0 \leq k \leq m-1 .
\end{aligned}
$$

Next, we estimate the error of interpolation $\mathcal{I}_{L, N, m, n} v$. Clearly $\mathcal{I}_{L, N, m, n} v(x)-$ $v(x)=\mathcal{I}_{N, m, n} \widetilde{v}(x)-\widetilde{v}(x)$. Thus, Lemma 3.2 implies the following result.

Theorem 3.1. If $v \in H_{m, n, A}^{\max (m, n)}(\Lambda), \partial_{x}^{r} v \in L_{\chi^{(-m+r,-n+r)}}^{2}(\Lambda)$, integers $m, n, r \geq$ $1, N \geq m+n$ and $0 \leq k \leq r \leq N+1$, then

$$
\begin{aligned}
& \left\|\partial_{x}^{k}\left(\mathcal{I}_{L, N, m, n} v-v\right)\right\|_{\chi^{(-m+k,-n+k)}}=\left\|\partial_{x}^{k}\left(\mathcal{I}_{N, m, n} \widetilde{v}-\widetilde{v}\right)\right\|_{\chi^{(-m+k,-n+k)}} \\
& \quad \leq c N^{k-r}\left(\left\|\partial_{x}^{r} v\right\|_{\chi^{(-m+r,-n+r)}}+\left\|\partial_{x}^{r} v_{m, n, b}\right\|_{\chi^{(-m+r,-n+r)}}\right) .
\end{aligned}
$$

Following the same line as the derivations of (2.21), (2.23) and (2.24), we obtain

$$
\begin{gathered}
\left\|\partial_{x}^{k}\left(\mathcal{I}_{L, N, m, n} v-v\right)\right\|_{\chi^{(-m+k,-n+k)}} \leq c N^{k-r}\left(\left\|\partial_{x}^{r} v\right\|_{\chi^{(-m+r,-n+r)}}\right. \\
\left.+\|v\|_{H^{\max (m, n)}(\Lambda)}\right), \text { for } \max (m, n) \leq r \leq m+n-1, \\
\left\|\partial_{x}^{k}\left(\mathcal{I}_{L, N, m, n} v-v\right)\right\|_{\chi^{(-m+k,-n+k)}} \leq c N^{k-r}\left\|\partial_{x}^{r} v\right\|_{\chi^{(-m+r,-n+r)}}, \\
\quad \text { for } 1 \leq m, n \leq 4, \max (m, n) \leq r \leq m+n-1 .
\end{gathered}
$$

Remark 3.1. If $m=n=1$, then the interpolation $\mathcal{I}_{L, N, m, n} v$ corresponds to a new interpolation $\mathcal{I}_{L, N, 1,1} v$. By (3.14) and (3.16), for $r \geq 1$, we get

$$
\left\|\partial_{x}^{k}\left(\mathcal{I}_{L, N, 1,1} v-v\right)\right\|_{\chi^{(-1+k,-1+k)}} \leq c N^{k-r}\left\|\partial_{x}^{r} v\right\|_{\chi^{(-1+r,-1+r)}}, \quad k=0,1 .
$$

The interpolation $\mathcal{I}_{L, N, 1,1} v$ is different from the standard Jacobi-Gauss-Labatto interpolation $\mathcal{I}_{L, N} v$ developed in [21. Moreover, it was only shown in Theorem 4.10 of [21] that $\left\|\partial_{x}^{k}\left(\mathcal{I}_{L, N} v-v\right)\right\| \leq c N^{2 k-r}\left\|\partial_{x}^{r} v\right\|_{\chi^{(-1+r,-1+r)}}$ for $k=0,1$.

Remark 3.2. If $m=n \geq 1$, then $\mathcal{I}_{L, N, m, m} v$ is equivalent to the generalized Legendre-Gauss-Labatto polynomial interpolation $k_{N}^{m} v$ for $v \in C_{m, n}^{\max (m-1, n-1)}(\bar{\Lambda})$, given by (13.17) of [3], since both of $\mathcal{I}_{L, N, m, n} v$ and $k_{N}^{m} v$ are polynomials of degree $N$, possess the same interpolation nodes and satisfy (3.12). The results (3.14) and (3.16) with $m=n=1, r \geq 1$ and $k=1$, was first given in [27]. On the other hand, Bernardi and Mady showed earlier that $\left\|k_{N}^{1} v-v\right\|_{\chi^{(-1,-1)}} \leq c N^{-r}\|v\|_{H^{r}(\Lambda)}, r \geq 1$; see (13.26) of [3]. Thus, (3.14) and (3.16) generalize and improve the corresponding results of [3, 27].

Remark 3.3. If (3.14) or (3.16) holds, then $\left\|\partial_{x}^{m}\left(\mathcal{I}_{L, N, m, m} v\right)\right\| \leq c\left\|\partial_{x}^{m} v\right\|$. Thus, the interpolation $\mathcal{I}_{L, N, m, m} v$ is stable in $H_{m, m, A}^{m}(\Lambda)$ !. The result with $m=1$ was given in $[3]$. 


\section{LAGUERRE QUASI-ORTHOGONAL APPROXIMATION}

4.1. Generalized Laguerre functions with arbitrary real parameter $\alpha$. Let $\Lambda=\{x \mid 0<x<\infty\}$ and $\chi(x)$ be a weight function. For integer $r \geq 0$, we define the weighted Sobolev space $H_{\chi}^{r}(\Lambda)$ as usual, with the inner product $(\cdot, \cdot)_{r, \chi}$, the semi-norm $|\cdot|_{r, \chi}$ and the norm $\|\cdot\|_{r, \chi}$. The inner product and the norm of $L_{\chi}^{2}(\Lambda)$ are denoted by $(\cdot, \cdot)_{\chi}$ and $\|\cdot\|_{\chi}$, respectively. Furthermore,

$$
{ }_{0} H_{\chi}^{r}(\Lambda)=\left\{v \in H_{\chi}^{r}(\Lambda) \mid \partial_{x}^{k} v(0)=0,0 \leq k \leq r-1\right\} .
$$

We omit the subscript $\chi$ whenever $\chi(x) \equiv 1$.

The scaled generalized Laguerre polynomial of degree $l \geq 0$ is defined by (cf. [28])

$$
L_{l}^{(\alpha, \beta)}(x)=\frac{1}{l !} x^{-\alpha} e^{\beta x} \partial_{x}^{l}\left(x^{l+\alpha} e^{-\beta x}\right), \quad \alpha>-1, \beta>0 .
$$

They satisfy the following relations:

$$
\begin{array}{ll}
L_{l}^{(\alpha, \beta)}(x)=L_{l}^{(\alpha+1, \beta)}(x)-L_{l-1}^{(\alpha+1, \beta)}(x), & l \geq 1, \\
\partial_{x} L_{l}^{(\alpha, \beta)}(x)=-\beta L_{l-1}^{(\alpha+1, \beta)}(x), & l \geq 1, \\
-x \partial_{x} L_{l}^{(\alpha, \beta)}(x)=(l+\alpha) L_{l-1}^{(\alpha, \beta)}(x)-l L_{l}^{(\alpha, \beta)}(x), & l \geq 1 .
\end{array}
$$

Let $\omega_{\alpha, \beta}(x)=x^{\alpha} e^{-\beta x}$. We have

$$
\int_{\Lambda} L_{l}^{(\alpha, \beta)}(x) L_{l^{\prime}}^{(\alpha, \beta)}(x) \omega_{\alpha, \beta}(x) d x=\gamma_{l}^{(\alpha, \beta)} \delta_{l, l^{\prime}}
$$

where $\gamma_{l}^{(\alpha, \beta)}=\frac{\Gamma(l+\alpha+1)}{\beta^{\alpha+1} l !}$.

We now turn to the generalized Laguerre functions with arbitrary real parameter $\alpha$. Denote by $[\alpha]$ the largest integer $\leq \alpha$. Let $\bar{l}_{\alpha}=[-\alpha]$ for $\alpha \leq-1$, and $\bar{l}_{\alpha}=0$ for $\alpha>-1$. Meanwhile, $l_{\alpha}=l-[-\alpha]$ for $\alpha \leq-1$, and $l_{\alpha}=l$ for $\alpha>-1$. The new generalized Laguerre function of degree $l$ is defined by

$$
\mathcal{L}_{l}^{(\alpha, \beta)}(x)= \begin{cases}x^{-\alpha} L_{l_{\alpha}}^{(-\alpha, \beta)}(x), & \alpha \leq-1, l \geq \bar{l}_{\alpha}=[-\alpha], \\ L_{l}^{(\alpha, \beta)}(x), & \alpha>-1, l \geq \bar{l}_{\alpha}=0 .\end{cases}
$$

If $\alpha>-1$ or $\alpha$ is a negative integer, then $\mathcal{L}_{l}^{(\alpha, \beta)}(x)$ turns out to be a polynomial of degree $l$.

The function $\mathcal{L}_{l}^{(\alpha, \beta)}(x)$ is the $l$ th eigenfunction of the following Sturm-Liouville equation:

$$
\partial_{x}\left(x^{\alpha+1} e^{-\beta x} \partial_{x} \mathcal{L}_{l}^{(\alpha, \beta)}(x)\right)+\lambda_{l}^{(\alpha, \beta)} x^{\alpha} e^{-\beta x} \mathcal{L}_{l}^{(\alpha, \beta)}(x)=0, \quad l \geq \bar{l}_{\alpha}
$$

with

$$
\lambda_{l}^{(\alpha, \beta)}= \begin{cases}\beta\left(l_{\alpha}-\alpha\right)=\beta(l-[-\alpha]-\alpha), & \text { for } \alpha \leq-1 \\ \beta l_{\alpha}=\beta l, & \text { for } \alpha>-1\end{cases}
$$
i.e.,

By virtue of (4.4), all $\mathcal{L}_{l}^{(\alpha, \beta)}(x)$ conform a complete $L_{\omega_{\alpha, \beta}}^{2}(\Lambda)$-orthogonal system,

$$
\int_{\Lambda} \mathcal{L}_{l}^{(\alpha, \beta)}(x) \mathcal{L}_{l^{\prime}}^{(\alpha, \beta)}(x) \omega_{\alpha, \beta}(x) d x=\eta_{l}^{(\alpha, \beta)} \delta_{l, l^{\prime}}
$$


where

$$
\eta_{l}^{(\alpha, \beta)}= \begin{cases}\gamma_{l_{\alpha}}^{(-\alpha, \beta)}=\gamma_{l-[-\alpha]}^{(-\alpha, \beta)}, & \text { for } \alpha \leq-1, l \geq \bar{l}_{\alpha}, \\ \gamma_{l}^{(\alpha, \beta)}, & \text { for } \alpha>-1, l \geq 0 .\end{cases}
$$

Thus, for any $v \in L_{\omega_{\alpha, \beta}}^{2}(\Lambda)$, we have

$$
v(x)=\sum_{l=\bar{l}_{\alpha}}^{\infty} \hat{v}_{l}^{(\alpha, \beta)} \mathcal{L}_{l}^{(\alpha, \beta)}(x)
$$

with

$$
\hat{v}_{l}^{(\alpha, \beta)}=\frac{1}{\eta_{l}^{(\alpha, \beta)}} \int_{\Lambda} v(x) \mathcal{L}_{l}^{(\alpha, \beta)}(x) \omega_{\alpha, \beta}(x) d x .
$$

Some important properties of $\mathcal{L}_{l}^{(\alpha, \beta)}(x)$ are stated in the following two propositions.

Proposition 4.1. We have

$$
\int_{\Lambda} \partial_{x} \mathcal{L}_{l}^{(\alpha, \beta)}(x) \partial_{x} \mathcal{L}_{l^{\prime}}^{(\alpha, \beta)}(x) \omega_{\alpha+1, \beta}(x) d x=\lambda_{l}^{(\alpha, \beta)} \eta_{l}^{(\alpha, \beta)} \delta_{l, l^{\prime}}, \quad l, l^{\prime} \geq \bar{l}_{\alpha} .
$$

Proof. We first consider $\alpha \leq-1$. In this case, $\bar{l}_{\alpha}=[-\alpha]$. By virtue of (4.5),

$$
x^{\alpha+1} \partial_{x} \mathcal{L}_{l}^{(\alpha, \beta)}(x)=-\alpha L_{l_{\alpha}}^{(-\alpha, \beta)}(x)+x \partial_{x} L_{l_{\alpha}}^{(-\alpha, \beta)}(x) .
$$

If $l \geq \bar{l}_{\alpha}+1$, then $l_{\alpha} \geq 1$. By using (4.3), we obtain from (4.13) that

$$
\begin{aligned}
x^{\alpha+1} \partial_{x} \mathcal{L}_{l}^{(\alpha, \beta)}(x) & =-\alpha L_{l_{\alpha}}^{(-\alpha, \beta)}(x)-\left(l_{\alpha}-\alpha\right) L_{l_{\alpha}-1}^{(-\alpha, \beta)}(x)+l_{\alpha} L_{l_{\alpha}}^{(-\alpha, \beta)}(x) \\
& =\left(l_{\alpha}-\alpha\right)\left(L_{l_{\alpha}}^{(-\alpha, \beta)}(x)-L_{l_{\alpha}-1}^{(-\alpha, \beta)}(x)\right) .
\end{aligned}
$$

The above with (4.1) leads to

$$
x^{\alpha+1} \partial_{x} \mathcal{L}_{l}^{(\alpha, \beta)}(x)=\left(l_{\alpha}-\alpha\right) L_{l_{\alpha}}^{(-\alpha-1, \beta)}(x) .
$$

If $l=\bar{l}_{\alpha}$, then $l_{\alpha}=0$. Accordingly, (4.13) leads to

$$
x^{\alpha+1} \partial_{x} \mathcal{L}_{l}^{(\alpha, \beta)}(x)=-\alpha L_{0}^{(-\alpha, \beta)}(x)+x \partial_{x} L_{0}^{(-\alpha, \beta)}(x)=-\alpha .
$$

Thus (4.14) is also valid. Further, by (2.2) of [22],

$$
L_{l_{\alpha}}^{(-\alpha-1, \beta)}(0)=\frac{\Gamma\left(l_{\alpha}-\alpha\right)}{\Gamma(-\alpha) \Gamma\left(l_{\alpha}+1\right)}=\frac{\Gamma(l-[-\alpha]-\alpha)}{\Gamma(-\alpha) \Gamma(l-[-\alpha]+1)}, \quad l_{\alpha} \geq 0 .
$$

Moreover, for any $v \in L_{\omega_{\alpha, \beta}}^{2}(\Lambda)$, we have $v=\mathrm{o}\left(x^{-\frac{\alpha+1}{2}}\right)$ as $x \rightarrow 0$. This fact, along with (4.14) and (4.15), implies that for $l \geq \bar{l}_{\alpha}$, we have $x^{\alpha+1} e^{-\beta x} \partial_{x} \mathcal{L}_{l}^{(\alpha, \beta)}(x) \mathcal{L}_{l^{\prime}}^{(\alpha, \beta)}(x)$ $\rightarrow 0$ as $x \rightarrow 0$. On the other hand, for any $v \in L_{\omega_{\alpha, \beta}}^{2}(\Lambda)$, we have $v=\mathrm{o}\left(x^{-\frac{\alpha+1}{2}} e^{\frac{1}{2} \beta x}\right)$ as $x \rightarrow \infty$. As a result, $x^{\alpha+1} e^{-\beta x} \partial_{x} \mathcal{L}_{l}^{(\alpha, \beta)}(x) \mathcal{L}_{l^{\prime}}^{(\alpha, \beta)}(x)$ also tends to zero as $x \rightarrow \infty$. Therefore, by multiplying both sides of $(4.6)$ by $\mathcal{L}_{l^{\prime}}^{(\alpha, \beta)}(x)$ and integrating the resulting equation by parts, we use (4.8) to obtain (4.12) with $\alpha \leq-1$.

Next, we consider $\alpha>-1$. Thanks to (4.5) and (4.2), we have $x^{\alpha+1} \partial_{x} \mathcal{L}_{l}^{(\alpha, \beta)}(x)=$ $-\beta x^{\alpha+1} L_{l-1}^{(\alpha+1, \beta)}(x)$ for all for $l \geq 1$. In this case, $x^{\alpha+1} e^{-\beta x} \partial_{x} \mathcal{L}_{l}^{(\alpha, \beta)}(x) \mathcal{L}_{l^{\prime}}^{(\alpha, \beta)}(x)$ $\rightarrow 0$ as $x \rightarrow 0, \infty$. Then, by the same argument as in the last part, we reach the desired result (4.12) with $l, l^{\prime} \geq 1$. Clearly, due to (4.7) with $\alpha>-1,(4.12)$ is also valid for $l, l^{\prime}=0$. 
Proposition 4.2. If $\alpha=-1$ or $\alpha \leq-2$, then

$$
\partial_{x} \mathcal{L}_{l}^{(\alpha, \beta)}(x)=(l-[-\alpha]-\alpha) \mathcal{L}_{l-1}^{(\alpha+1, \beta)}(x), \quad l \geq \bar{l}_{\alpha} .
$$

Proof. We have from (4.14) that for $\alpha \leq-1$,

$$
\partial_{x} \mathcal{L}_{l}^{(\alpha, \beta)}(x)=\left(l_{\alpha}-\alpha\right) x^{-\alpha-1} L_{l-[-\alpha]}^{(-\alpha-1, \beta)}(x), \quad l \geq \bar{l}_{\alpha} .
$$

If $\alpha \leq-2$, then by using (4.5), we obtain from (4.17) that

$$
\partial_{x} \mathcal{L}_{l}^{(\alpha, \beta)}(x)=\left(l_{\alpha}-\alpha\right) \mathcal{L}_{l-1}^{(\alpha+1, \beta)}(x)=(l-[-\alpha]-\alpha) \mathcal{L}_{l-1}^{(\alpha+1, \beta)}(x), \quad l \geq \bar{l}_{\alpha} .
$$

If $\alpha=-1$, then $l_{\alpha}=l-1, l_{\alpha}-\alpha=l$ and $L_{l_{\alpha}}^{(-\alpha-1, \beta)}(x)=L_{l-1}^{(0, \beta)}(x)$. Thereby, we have from (4.17) and (4.5) that $\partial_{x} \mathcal{L}_{l}^{(-1, \beta)}(x)=l L_{l-1}^{(0, \beta)}(x)=l \mathcal{L}_{l-1}^{(0, \beta)}(x)$. This is the desired result (4.16) with $\alpha=-1$. The proof is completed.

\subsection{Orthogonal approximation with arbitrary real parameter $\alpha$. Let}

$$
Q_{N}^{(\alpha, \beta)}(\Lambda)=\operatorname{span}\left\{\mathcal{L}_{l}^{(\alpha, \beta)}(x), \bar{l}_{\alpha} \leq l \leq N\right\}
$$

The orthogonal projection $P_{N, \alpha, \beta}: L_{\omega_{\alpha, \beta}}^{2}(\Lambda) \rightarrow Q_{N}^{(\alpha, \beta)}(\Lambda)$ is defined by

$$
\left(P_{N, \alpha, \beta} v-v, \phi\right)_{\omega_{\alpha, \beta}}=0, \quad \forall \phi \in Q_{N}^{(\alpha, \beta)}(\Lambda) .
$$

For estimation of approximation error, we introduce the Sturm-Liouville operator as

$$
\mathcal{A}_{\alpha, \beta} v(x)=-x^{-\alpha} e^{\beta x} \partial_{x}\left(x^{\alpha+1} e^{-\beta x} \partial_{x} v(x)\right) .
$$

Evidently, by (4.6),

$$
\mathcal{A}_{\alpha, \beta} \mathcal{L}_{l}^{(\alpha, \beta)}(x)=\lambda_{l}^{(\alpha, \beta)} \mathcal{L}_{l}^{(\alpha, \beta)}(x), \quad l \geq \bar{l}_{\alpha} .
$$

Moreover, an argument similar to Proposition 4.1, shows that for any $v \in L_{\omega_{\alpha, \beta}}^{2}(\Lambda)$,

$$
x^{\alpha+1} e^{-\beta x} v(x) \partial_{x} \mathcal{L}_{l}^{(\alpha, \beta)}(x) \rightarrow 0, \quad \text { as } x \rightarrow 0, \infty .
$$

Therefore, by (4.18) and integration by parts, we obtain

$$
\left(v, \mathcal{L}_{l}^{(\alpha, \beta)}\right)_{\omega_{\alpha, \beta}}=\left(\lambda_{l}^{(\alpha, \beta)}\right)^{-1}\left(v, \mathcal{A}_{\alpha, \beta} \mathcal{L}_{l}^{(\alpha, \beta)}\right)_{\omega_{\alpha, \beta}}=\left(\lambda_{l}^{(\alpha, \beta)}\right)^{-1}\left(\mathcal{A}_{\alpha, \beta} v, \mathcal{L}_{l}^{(\alpha, \beta)}\right)_{\omega_{\alpha, \beta}} .
$$

Therefore, if $u, v$ are in the domain of the operator $\mathcal{A}_{\alpha, \beta}$, then $\left(\mathcal{A}_{\alpha, \beta} u, v\right)_{\omega_{\alpha, \beta}}=$ $\left(u, \mathcal{A}_{\alpha, \beta} v\right)_{\omega_{\alpha, \beta}}$. Accordingly, $\mathcal{A}_{\alpha, \beta}$ is a positive definite and self-conjugate operator. Thus, we could define the following Sobolev-type spaces with integer $r \geq 0$,

$$
\begin{gathered}
D\left(\mathcal{A}_{\alpha, \beta}^{r}\right)=\left\{v \mid \mathcal{A}_{\alpha, \beta}^{k} v \in L_{\omega_{\alpha, \beta}}^{2}(\Lambda), 0 \leq k \leq r\right\} \\
D\left(\mathcal{A}_{\alpha, \beta}^{r+\frac{1}{2}}\right)=\left\{v \mid v \in D\left(\mathcal{A}_{\alpha, \beta}^{r}\right) \text { and } \partial_{x} \mathcal{A}_{\alpha, \beta}^{r} v \in L_{\omega_{\alpha+1, \beta}}^{2}(\Lambda)\right\},
\end{gathered}
$$

equipped with the following semi-norms and norms,

$$
\begin{array}{rlrl}
|v|_{D\left(\mathcal{A}_{\alpha, \beta}^{r}\right)} & =\left\|\mathcal{A}_{\alpha, \beta}^{r} v\right\|_{\omega_{\alpha, \beta},} & |v|_{D\left(\mathcal{A}_{\alpha, \beta}^{r+\frac{1}{2}}\right)}=\left\|\partial_{x} \mathcal{A}_{\alpha, \beta}^{r} v\right\|_{\omega_{\alpha+1, \beta}}, \\
\|v\|_{D\left(\mathcal{A}_{\alpha, \beta}^{r}\right)}=\left(\sum_{k=0}^{r}|v|_{D\left(\mathcal{A}_{\alpha, \beta}^{k}\right)}^{2}\right)^{\frac{1}{2}}, & \|v\|_{D\left(\mathcal{A}_{\alpha, \beta}^{r+1 / 2}\right)}=\left(\|v\|_{D\left(\mathcal{A}_{\alpha, \beta}^{r}\right)}^{2}+|v|_{D\left(\mathcal{A}_{\alpha, \beta}^{r+1 / 2}\right)}^{2}\right)^{\frac{1}{2}} .
\end{array}
$$

Theorem 4.1. For any $v \in D\left(\mathcal{A}_{\alpha, \beta}^{\frac{r}{2}}\right)$ and integers $0 \leq \mu \leq r \leq N+1$,

$$
\left|P_{N, \alpha, \beta} v-v\right|_{D\left(\mathcal{A}_{\alpha, \beta}^{\frac{\mu}{2}}\right)} \leq c(\beta N)^{\frac{\mu-r}{2}}|v|_{D\left(\mathcal{A}_{\alpha, \beta}^{\frac{r}{2}}\right)} .
$$


Proof. We first consider even integers $\mu$. In this case, we use (4.10), (4.18), (4.8) and (4.11) successively to obtain

$$
\left|P_{N, \alpha, \beta} v-v\right|_{D\left(\mathcal{A}_{\alpha, \beta}^{\frac{\mu}{2}}\right)}^{2}=\sum_{l=N+1}^{\infty}\left(\lambda_{l}^{(\alpha, \beta)}\right)^{\mu}\left(\eta_{l}^{(\alpha, \beta)}\right)^{-1}\left(v, \mathcal{L}_{l}^{(\alpha, \beta)}\right)_{\omega_{\alpha, \beta}}^{2} .
$$

For even integer $r=2 q$, we use (4.18) and (4.20) to obtain

$$
\left(v, \mathcal{L}_{l}^{(\alpha, \beta)}\right)_{\omega_{\alpha, \beta}}=\left(\lambda_{l}^{(\alpha, \beta)}\right)^{-q}\left(\mathcal{A}_{\alpha, \beta}^{q} v, \mathcal{L}_{l}^{(\alpha, \beta)}\right)_{\omega_{\alpha, \beta}} .
$$

A combination of the above two equalities with (4.7) yields

$$
\begin{aligned}
\left|P_{N, \alpha, \beta} v-v\right|_{D\left(A_{\alpha, \beta}^{\frac{\mu}{2}}\right)}^{2} & \leq c\left(\lambda_{N+1}^{(\alpha, \beta)}\right)^{\mu-2 q} \sum_{l=N+1}^{\infty}\left(\eta_{l}^{(\alpha, \beta)}\right)^{-1}\left(\mathcal{A}_{\alpha, \beta}^{q} v, \mathcal{L}_{l}^{(\alpha, \beta)}\right)_{\omega_{\alpha, \beta}}^{2} \\
& \leq c(\beta N)^{\mu-r} \sum_{l=\bar{l}_{\alpha}}^{\infty}\left(\eta_{l}^{(\alpha, \beta)}\right)^{-1}\left(\mathcal{A}_{\alpha, \beta}^{q} v, \mathcal{L}_{l}^{(\alpha, \beta)}\right)_{\omega_{\alpha, \beta}}^{2}
\end{aligned}
$$

Furthermore, thanks to (4.8), (4.10) and (4.11), we have

$$
\left\|\mathcal{A}_{\alpha, \beta}^{q} v\right\|_{\omega_{\alpha, \beta}}^{2}=\sum_{l=\bar{l}_{\alpha}}^{\infty}\left(\eta_{l}^{(\alpha, \beta)}\right)^{-1}\left(\mathcal{A}_{\alpha, \beta}^{q} v, \mathcal{L}_{l}^{(\alpha, \beta)}\right)_{\omega_{\alpha, \beta}}^{2} .
$$

Therefore,

$$
\left|P_{N, \alpha, \beta} v-v\right|_{D\left(\mathcal{A}_{\alpha, \beta}^{\frac{\mu}{2}}\right)}^{2} \leq c(\beta N)^{\mu-r}\left\|\mathcal{A}_{\alpha, \beta}^{q} v\right\|_{\omega_{\alpha, \beta}}^{2}=c(\beta N)^{\mu-r}|v|_{D\left(\mathcal{A}_{\alpha, \beta}^{2}\right)}^{2} .
$$

Next, we consider odd integer $r=2 q+1$. By (4.6), (4.19) and integration by parts, we obtain from (4.22) that

$$
\left(v, \mathcal{L}_{l}^{(\alpha, \beta)}\right)_{\omega_{\alpha, \beta}}=\left(\lambda_{l}^{(\alpha, \beta)}\right)^{-q-1}\left(\partial_{x} \mathcal{A}_{\alpha, \beta}^{q} v, \partial_{x} \mathcal{L}_{l}^{(\alpha, \beta)}\right)_{\omega_{\alpha+1, \beta}}
$$

Accordingly, we use (4.10)-(4.12) and (4.18) successively to verify that

$$
\begin{aligned}
& \left|P_{N, \alpha, \beta} v-v\right|_{D\left(\mathcal{A}_{\alpha, \beta}^{\frac{\mu}{2}}\right)}^{2}=\sum_{l=N+1}^{\infty}\left(\lambda_{l}^{(\alpha, \beta)}\right)^{\mu}\left(\eta_{l}^{(\alpha, \beta)}\right)^{-1}\left(v, \mathcal{L}_{l}^{(\alpha, \beta)}\right)_{\omega_{\alpha, \beta}}^{2} \\
& \quad \leq c\left(\lambda_{N+1}^{(\alpha, \beta)}\right)^{\mu-2 q-1} \sum_{l=N+1}^{\infty}\left(\lambda_{l}^{(\alpha, \beta)}\right)^{-1}\left(\eta_{l}^{(\alpha, \beta)}\right)^{-1}\left(\partial_{x} \mathcal{A}_{\alpha, \beta}^{q} v, \partial_{x} \mathcal{L}_{l}^{(\alpha, \beta)}\right)_{\omega_{\alpha+1, \beta}} \\
& \quad \leq c(\beta N)^{\mu-r}\left\|\partial_{x} \mathcal{A}_{\alpha, \beta}^{q} v\right\|_{\omega_{\alpha+1, \beta}}=c(\beta N)^{\mu-r}|v|_{D\left(\mathcal{A}_{\alpha, \beta}^{\frac{r}{2}}\right)}^{2}
\end{aligned}
$$

We can deal with the case with odd integer $\mu$ in the same manner as before.

4.3. Laguerre orthogonal approximation with negative integer $\alpha=-m$. In the sequel, we assume integer $m \geq 1$. Clearly, $\mathcal{L}_{l}^{(-m, \beta)}(x)=x^{m} L_{l-m}^{(m, \beta)}(x)$, $l \geq m$, and

$$
\partial_{x}^{k} \mathcal{L}_{l}^{(-m, \beta)}(0)=0, \quad 0 \leq k \leq m-1 .
$$

We shall show that for integers $m \geq 1$ and $k \geq 0$,

$$
\partial_{x}^{k} \mathcal{L}_{l}^{(-m, \beta)}(x)=D_{k, l}^{m, \beta} \mathcal{L}_{l-k}^{(-m+k, \beta)}(x), \quad l \geq \max (m, k),
$$


where $D_{0, l}^{m, \beta}=1$ and

$$
D_{k, l}^{m, \beta}= \begin{cases}\prod_{j=0}^{k-1}(l-j), & \text { if } 1 \leq k \leq m, \\ (-\beta)^{k-m} \prod_{j=0}^{m-1}(l-j), & \text { if } 1 \leq m<k .\end{cases}
$$

In fact, we could use (4.16) to obtain the result (4.24) with $k \leq m \leq l$ inductively. For $m<k \leq l$, we use (4.16) and (4.2) repeatedly to deduce that

$$
\begin{aligned}
\partial_{x}^{k} \mathcal{L}_{l}^{(-m, \beta)}(x)=\prod_{j=0}^{m-1}(l-j) \partial_{x}^{k-m} \mathcal{L}_{l-m}^{(0, \beta)}(x) \\
\quad=\prod_{j=0}^{m-1}(l-j) \partial_{x}^{k-m} L_{l-m}^{(0, \beta)}(x)=(-\beta)^{k-m} \prod_{j=0}^{m-1}(l-j) L_{l-k}^{(-m+k, \beta)}(x) \\
\quad=(-\beta)^{k-m} \prod_{j=0}^{m-1}(l-j) \mathcal{L}_{l-k}^{(-m+k, \beta)}(x) .
\end{aligned}
$$

A combination of (4.24) and (4.8) with $\alpha=-m$ leads to

$$
\int_{\Lambda} \partial_{x}^{k} \mathcal{L}_{l}^{(-m, \beta)}(x) \partial_{x}^{k} \mathcal{L}_{l^{\prime}}^{(-m, \beta)}(x) \omega_{-m+k, \beta}(x) d x=\left(D_{k, l}^{m, \beta}\right)^{2} \eta_{l-k}^{(-m+k, \beta)} \delta_{l, l^{\prime}} .
$$

In the above equality, there exists the factor $\eta_{l-k}^{(-m+k, \beta)}$. We now estimate its upper bound. Actually, by virtue of (4.9) with $\alpha=-m$ and (4.4),

$$
\eta_{l}^{(-m, \beta)}=\gamma_{l-m}^{(m, \beta)}=\frac{l !}{\beta^{m+1}(l-m) !}, \quad \text { for } l \geq m .
$$

Hence, using (4.4) again yields

$$
\eta_{l-k}^{(-m+k, \beta)}=\gamma_{l-m}^{(m-k, \beta)}=\frac{(l-k) !}{\beta^{m-k+1}(l-m) !}, \quad \text { for } m \geq k+1 .
$$

Similarly,

$$
\eta_{l-k}^{(-m+k, \beta)}=\gamma_{l-k}^{(-m+k, \beta)}=\frac{(l-m) !}{\beta^{k-m+1}(l-k) !}, \quad \quad \text { for } m<k+1 .
$$

Now, $\mathcal{P}_{N}(\Lambda)$ stands for the set of all algebraic polynomials of degree at most $N$, and

$$
\mathcal{P}_{N}^{m, 0}(\Lambda)=\left\{\phi \in \mathcal{P}_{N}(\Lambda) \mid \partial_{x}^{k} \phi(0)=0, \text { for } 0 \leq k \leq m-1\right\} .
$$

Obviously,

$$
Q_{N}^{(-m, \beta)}(\Lambda)=\operatorname{span}\left\{\mathcal{L}_{m}^{(-m, \beta)}(x), \mathcal{L}_{m+1}^{(-m, \beta)}(x), \cdots, \mathcal{L}_{N}^{(-m, \beta)}(x)\right\}=\mathcal{P}_{N}^{m, 0}(\Lambda) .
$$

The following inverse inequality will be used in the next section.

Proposition 4.3. For any $\phi \in Q_{N}^{(-m, \beta)}(\Lambda)$ with $m \geq 1$,

$$
\left\|\partial_{x}^{k} \phi\right\|_{\omega_{-m+k, \beta}} \leq c(\beta N)^{\frac{k}{2}}\|\phi\|_{\omega_{-m, \beta}} .
$$

Proof. We use (4.24) and (4.26) to obtain

$$
\left\|\partial_{x}^{k} \phi\right\|_{\omega_{-m+k, \beta}}^{2}=\sum_{l=\max (m, k)}^{N}\left(\hat{\phi}_{l}^{(-m, \beta)}\right)^{2}\left(D_{k, l}^{m, \beta}\right)^{2} \eta_{l-k}^{(-m+k, \beta)} .
$$


Let $C_{N, k}^{(m, \beta)}=\max _{m \leq l \leq N} \frac{\left(D_{k, l}^{m, \beta}\right)^{2} \eta_{l-k}^{(-m+k, \beta)}}{\eta_{l}^{(-m, \beta)}}$. We use (4.25), (4.28) and (4.39) to verify that for large $l$,

$$
D_{k, l}^{m, \beta} \sim l^{k}, \text { if } m \geq k, \quad D_{k, l}^{m, \beta} \sim \beta^{k-m} l^{m} \text {, if } m<k,
$$

while

$$
\eta_{l-k}^{(-m+k, \beta)} \sim l^{m-k} \beta^{k-m-1}, \text { if } m \geq k, \quad \eta_{l-k}^{(-m+k, \beta)} \sim l^{k-m} \beta^{m-k-1} \text {, if } m<k .
$$

Thereby,

$$
\left(D_{k, l}^{m, \beta}\right)^{2} \eta_{l-k}^{(-m+k, \beta)} \leq c l^{k+m} \beta^{k-m-1} .
$$

In addition, $\eta_{l}^{(-m, \beta)} \sim \frac{1}{\beta^{m+1}} l^{m}$. Consequently, $C_{N, k}^{(m, \beta)} \leq c(\beta N)^{k}$. Finally, we obtain from (4.31) that

$$
\left\|\partial_{x}^{k} \phi\right\|_{\omega_{-m+k, \beta}}^{2} \leq C_{N, k}^{(m, \beta)} \sum_{l=\max (m, k)}^{N}\left(\hat{\phi}_{l}^{(-m, \beta)}\right)^{2} \eta_{l}^{(-m, \beta)} \leq c(\beta N)^{k}\|\phi\|_{\omega_{-m, \beta}}^{2} .
$$

This ends the proof.

We now state the main result of this subsection.

Theorem 4.2. If $\partial_{x}^{k} v \in L_{\omega_{-m+k, \beta}}^{2}(\Lambda), \partial_{x}^{r} v \in L_{\omega_{-m+r, \beta}}^{2}(\Lambda)$, integers $1 \leq m \leq$ $\min (r, N)$, and $0 \leq k \leq r \leq N+1$, then

$$
\left\|\partial_{x}^{k}\left(P_{N,-m, \beta} v-v\right)\right\|_{\omega_{-m+k, \beta}} \leq c(\beta N)^{\frac{k-r}{2}}\left\|\partial_{x}^{r} v\right\|_{\omega_{-m+r, \beta}} .
$$

Proof. We use (4.10) and (4.24) to obtain

$$
\partial_{x}^{k}\left(P_{N,-m, \beta} v-v\right)=-\sum_{l=N+1}^{\infty} D_{k, l}^{m, \beta} \hat{v}_{l}^{(-m, \beta)} \mathcal{L}_{l-k}^{(-m+k, \beta)}(x) .
$$

This with (4.26) implies

$$
\left\|\partial_{x}^{k}\left(P_{N,-m, \beta} v-v\right)\right\|_{\omega_{-m+k, \beta}}^{2}=\sum_{l=N+1}^{\infty}\left(D_{k, l}^{m, \beta}\right)^{2} \eta_{l-k}^{(-m+k, \beta)}\left(\hat{v}_{l}^{(-m, \beta)}\right)^{2} .
$$

On the other hand, since $Q_{N}^{(-m, \beta)}(\Lambda)=\mathcal{P}_{N}^{m, 0}(\Lambda)$, we use (4.26) to obtain

$$
\left\|\partial_{x}^{r} v\right\|_{\omega_{-m+r, \beta}}^{2}=\sum_{l=r}^{\infty}\left(D_{r, l}^{m, \beta}\right)^{2} \eta_{l-r}^{(-m+r, \beta)}\left(\hat{v}_{l}^{(-m, \beta)}\right)^{2} .
$$

Let $C_{N, k, r}^{(m, \beta)}=\max _{l>N} \frac{\left(D_{k, l}^{m, \beta}\right)^{2} \eta_{l-k}^{(-m+k, \beta)}}{\left(D_{r, l}^{m, \beta}\right)^{2} \eta_{l-r}^{(-m+r, \beta)}}$. Due to $(4.32)$, we have $C_{N, k, r}^{(m, \beta)} \leq c(\beta N)^{k-r}$. Accordingly, $\left\|\partial_{x}^{k}\left(P_{N,-m, \beta} v-v\right)\right\|_{\omega_{-m+k, \beta}}^{2} \leq c(\beta N)^{k-r}\left\|\partial_{x}^{r} v\right\|_{\omega_{-m+r, \beta}}^{2}$. This ends the proof.

In numerical analysis, we need other orthogonal projections. For this purpose, we introduce the following space with integer $r \geq 0$,

$$
H_{\omega_{-m, \beta}, A}^{r}(\Lambda)=\left\{v \mid v \text { is measurable on } \Lambda \text { and }\|v\|_{H_{\omega_{-m, \beta}, A}^{r}<\infty}\right\},
$$

equipped with the semi-norm and the norm as

$$
|v|_{H_{\omega_{-m, \beta}, A}^{r}}=\left\|\partial_{x}^{r} v\right\|_{\omega_{-m+r, \beta}}, \quad\|v\|_{H_{\omega_{-m, \beta}, A}^{r}}=\left(\sum_{k=0}^{r}\left\|\partial_{x}^{k} v\right\|_{\omega_{-m+k, \beta}}^{2}\right)^{\frac{1}{2}} .
$$


Moreover, for $1 \leq m \leq r$,

$$
\begin{aligned}
{ }_{0} H_{\omega_{-m, \beta}, A}^{r}(\Lambda)= & \left\{v \mid v \in H_{\omega_{-m, \beta}, A}^{r}(\Lambda) \text { and } \partial_{x}^{k} v(0)=0, \text { for } 0 \leq k \leq r-1\right\}, \\
& B_{m, \beta}^{r}(\Lambda)={ }_{0} H_{\omega_{-m, \beta}, A}^{m}(\Lambda) \cap H_{\omega_{-m, \beta}, A}^{r}(\Lambda) .
\end{aligned}
$$
by

For integers $m \geq 1$, the projection ${ }_{0} P_{N,-m, \beta}^{m}: B_{m, \beta}^{m}(\Lambda) \rightarrow Q_{N}^{(-m, \beta)}(\Lambda)$ is defined

$$
\left(\partial_{x}^{m}\left(v-{ }_{0} P_{N,-m, \beta}^{m} v\right), \partial_{x}^{m} \phi\right) \omega_{\omega_{0, \beta}}=0, \quad \forall \phi \in Q_{N}^{(-m, \beta)}(\Lambda) .
$$

There is a close relation between ${ }_{0} P_{N,-m, \beta}^{m} v$ and $P_{N,-m, \beta} v$. To shows this, we assume $v \in B_{m, \beta}^{m}(\Lambda)$, and

$$
{ }_{0} P_{N,-m, \beta}^{m} v(x)=\sum_{l=m}^{N} a_{l}^{(-m, \beta)} \mathcal{L}_{l}^{(-m, \beta)}(x) .
$$

Thanks to (4.24), we have

$$
\begin{aligned}
\partial_{x}^{m}\left(v-{ }_{0} P_{N,-m, \beta}^{m} v\right)= & \sum_{l=m}^{N} D_{m, l}^{m, \beta}\left(\hat{v}_{l}^{(-m, \beta)}-a_{l}^{(-m, \beta)}\right) \mathcal{L}_{l-m}^{(0, \beta)}(x) \\
& +\sum_{l=N+1}^{\infty} D_{m, l}^{m, \beta} \hat{v}_{l}^{(-m, \beta)} \mathcal{L}_{l-m}^{(0, \beta)}(x) .
\end{aligned}
$$

Inserting the above equality into (4.24) with $\phi=\mathcal{L}_{l^{\prime}}^{(-m, \beta)}(x), m \leq l^{\prime} \leq N$, we use (4.24) and (4.26) to obtain

$$
\left(D_{m, l}^{m, \beta}\right)^{2} \eta_{l-m}^{(0, \beta)}\left(\hat{v}_{l}^{(-m, \beta)}-a_{l}^{(-m, \beta)}\right)^{2}=0, \quad \forall m \leq l \leq N .
$$

This means ${ }_{0} P_{N,-m, \beta}^{m} v(x)=P_{N,-m, \beta} v(x)$ for $v \in B_{m, \beta}^{m}(\Lambda)$.

The above matter with (4.33) leads to the following conclusion.

Theorem 4.3. If $v \in B_{m, \beta}^{m}(\Lambda), \partial_{x}^{r} v \in L_{\omega_{-m+r, \beta}}^{2}(\Lambda)$, integers $1 \leq m \leq \min (r, N)$, $0 \leq k \leq r \leq N+1$ and $k \leq m$, then

$$
\left\|\partial_{x}^{k}\left({ }_{0} P_{N,-m, \beta}^{m} v-v\right)\right\|_{\omega_{-m+k, \beta}} \leq c(\beta N)^{\frac{k-r}{2}}\left\|\partial_{x}^{r} v\right\|_{\omega_{-m+r, \beta}} .
$$

Remark 4.1. The above result is available for high order problems. But, the releted earlier results are only applicable to second or fourth order problems; see [3, 6, 10, 17, 28. It also improves the existing results essentially. For example, the estimate (4.35) with $\mu=m=1$ and $k=0$ implies the optimal error estimate, namely, $\left\|_{0} P_{N,-1, \beta}^{1} v-v\right\|_{\omega_{-1, \beta}} \leq c(\beta N)^{\frac{-r}{2}}\left\|\partial_{x}^{r} v\right\|_{\omega_{-1+r, \beta}}$. However, it was only shown before that (cf. Theorem 2.3 of [28]) $\left\|_{0} P_{N, \alpha, \beta}^{1} v-v\right\|_{\omega_{\alpha, \beta}} \leq c(\beta N)^{\frac{1-r}{2}}\left\|\partial_{x}^{r} v\right\|_{\omega_{\alpha-1+r, \beta}}$ for $|\alpha|<1$. Indeed, how to derive the above optimal error estimate had been an open problem for several decades.

Remark 4.2. Everitt, Littlejohn and Wellman 9] also considered the case with integer $\alpha \leq-1$, without error estimate. 
4.4. Laguerre quasi-orthogonal approximation. We set

$$
v_{b, m}(x)=\sum_{j=0}^{m-1} \partial_{x}^{j} v(0) \frac{x^{j}}{j !} .
$$

For any $v \in H_{\omega_{-m, \beta}, A}^{m}(\Lambda)$, we set $\tilde{v}(x)=v(x)-v_{b, m}(x)$. Since $\tilde{v}(x) \in B_{m, \beta}^{m}(\Lambda)$, we define the Laguerre quasi-orthogonal projection as

$$
P_{N,-m, \beta}^{m} v(x)={ }_{0} P_{N,-m, \beta}^{m} \tilde{v}(x)+v_{b, m}(x) \in \mathcal{P}_{N}(\Lambda) .
$$

Obviously, $\partial_{x}^{k} P_{N,-m, \beta}^{m} v(0)=\partial_{x}^{k} v(0)$ for $0 \leq k \leq m-1$. Moreover, $P_{N,-m, \beta}^{m} v(x)-$ $v(x)={ }_{0} P_{N,-m, \beta} \tilde{v}(x)-\tilde{v}(x)$, and $\partial_{x}^{r} v_{b, m}(x)=0$ for $r \geq m$. Thus, we use (4.35) to derive the following result.

Theorem 4.4. If $v \in H_{\omega_{-m, \beta}, A}^{m}(\Lambda), \partial_{x}^{r} v \in L_{\omega_{-m+r, \beta}}^{2}(\Lambda)$, integers $1 \leq m \leq$ $\min (r, N), 0 \leq k \leq r \leq N+1$ and $k \leq m$, then

$$
\left\|\partial_{x}^{k}\left(P_{N,-m, \beta}^{m} v-v\right)\right\|_{\omega_{-m+k, \beta}} \leq c(\beta N)^{\frac{k-r}{2}}\left\|\partial_{x}^{r} v\right\|_{\omega_{-m+r, \beta}} .
$$

\section{Generalized Laguerre-Gauss-Radau interpolation}

In this section, we investigate the generalized Laguerre-Gauss-Radau interpolation.

For $\alpha>-1$ and $\beta>0$, we denote by $\xi_{G, N, j}^{(\alpha, \beta)}(0 \leq j \leq N)$ the zeros of the polynomial $L_{N+1}^{(\alpha, \beta)}(x)$, which are arranged in ascending order. Meanwhile, $\omega_{G, N, j}^{(\alpha, \beta)}(0 \leq j \leq$ $N)$ stand for the corresponding Christoffel numbers such that

$$
\int_{\Lambda} \phi(x) \omega_{\alpha, \beta}(x) d x=\sum_{j=0}^{N} \phi\left(\xi_{G, N, j}^{(\alpha, \beta)}\right) \omega_{G, N, j}^{(\alpha, \beta)}, \quad \forall \phi \in \mathcal{P}_{2 N+1}(\Lambda) .
$$

We know from (2.10) of [22] that

$$
\omega_{G, N, j}^{(\alpha, \beta)}=\frac{\Gamma(N+\alpha+2)}{\beta^{\alpha} \Gamma(N+2)} \cdot \frac{1}{\xi_{G, N, j}^{(\alpha, \beta)}\left[\partial_{x} L_{N+1}^{(\alpha, \beta)}\left(\xi_{G, N, j}^{(\alpha, \beta)}\right]^{2}\right.}, \quad 0 \leq j \leq N .
$$

Let $\xi_{G, N,-1}^{(\alpha, \beta)}=0$. According to $(2.15)-(2.19)$ of [22], there exists a certain fixed number $\eta>0$, and the constants $c_{1} \sim \frac{\pi^{2}}{2}$ and $c_{2} \sim 8$, such that

- $2 \beta^{\frac{1}{2}}\left(\xi_{G, N, j}^{(\alpha, \beta)}\right)^{\frac{1}{2}}=\frac{1}{\sqrt{N+1}}(j \pi+\mathcal{O}(1))$, for $0<\xi_{G, N, j}^{(\alpha, \beta)} \leq \frac{\eta}{\beta}$,

- $\frac{c_{1} j^{2}}{\beta(2 N+\alpha+3)}<\xi_{G, N, j}^{(\alpha, \beta)}<\frac{c_{2} j^{2}}{\beta(2 N+\alpha+3)}$, for large $j$,

- $\xi_{G, N, N}^{(\alpha, \beta)}<4 \beta^{-1}(N+1)$,

- $\omega_{G, N, j}^{(\alpha, \beta)} \sim \frac{\pi}{\sqrt{\beta N}} e^{-\beta \xi_{G, N, j}^{(\alpha, \beta)}}\left(\xi_{G, N, j}^{(\alpha, \beta)}\right)^{\alpha+\frac{1}{2}}$, for $0<\xi_{G, N, j}^{(\alpha, \beta)} \leq \frac{\eta}{\beta}$,

- $\omega_{G, N, j}^{(\alpha, \beta)} \sim \omega_{\alpha, \beta}\left(\xi_{G, N, j}^{(\alpha, \beta)}\right)\left(\xi_{G, N, j}^{(\alpha, \beta)}-\xi_{G, N, j-1}^{(\alpha, \beta)}\right)$, for $0 \leq j \leq N$.

Now, let $m \geq 0$, and

$$
\xi_{N, j}^{(m, \beta)}=\xi_{G, N-m, j}^{(m, \beta)}, \quad \omega_{N, j}^{(m, \beta)}=\omega_{G, N-m, j}^{(m, \beta)}\left(\xi_{G, N-m, j}^{(m, \beta)}\right)^{-2 m}, 0 \leq j \leq N-m .
$$


We set $\xi_{N,-1}^{(m, \beta)}=0$. Then the previous statements lead to the fact that

$$
\begin{aligned}
& 2 \beta^{\frac{1}{2}}\left(\xi_{N, j}^{(m, \beta)}\right)^{\frac{1}{2}}=\frac{1}{\sqrt{N-m+1}}(j \pi+O(1)), \quad 0<\xi_{N, j}^{(m, \beta)} \leq \frac{\eta}{\beta}, \\
& \frac{c_{1} j^{2}}{\beta(2 N-m+3)}<\xi_{N, j}^{(m, \beta)}<\frac{c_{2} j^{2}}{\beta(2 N-m+3)}, \quad \text { for large } j, \\
& \omega_{N, j}^{(m, \beta)} \sim \frac{\pi}{\sqrt{\beta(N-m-m}} e^{-\beta \xi_{N, j}^{(m, \beta)}}\left(\xi_{N, j}^{(m, \beta)}\right)^{-m+\frac{1}{2}}, \quad 0<\xi_{N, j}^{(m, \beta)} \leq \frac{\eta}{\beta}, \\
& \omega_{N, j}^{(m, \beta)} \sim \omega_{-m, \beta}\left(\xi_{N, j}^{(m, \beta)}\right)\left(\xi_{N, j}^{(m, \beta)}-\xi_{N, j-1}^{(m, \beta)}\right), \quad 0 \leq j \leq N-m .
\end{aligned}
$$

Next, we introduce the discrete inner product and norm as follows:

$$
(u, v)_{N, m, \beta}=\sum_{j=0}^{N-m} u\left(\xi_{N, j}^{(m, \beta)}\right) v\left(\xi_{N, j}^{(m, \beta)}\right) \omega_{N, j}^{(m, \beta)}, \quad\|u\|_{N, m, \beta}=(u, u)_{N, m, \beta}^{\frac{1}{2}} .
$$

We can use (5.1) to show that

$$
(\phi, \psi)_{\omega_{-m, \beta}}=(\phi, \psi)_{N, m, \beta}, \quad \forall \phi \in Q_{N}^{(-m, \beta)}(\Lambda), \psi \in Q_{N+1}^{(-m, \beta)}(\Lambda) .
$$

For any integer $r \geq 0$, we denote by $C^{r}(\Lambda)$ the space consisting of all $r$-times differential functions. Further, for integer $r \geq m-1$,

$$
C_{0, m, \beta}^{r}(\Lambda)=\left\{v \in C^{r}(\Lambda) \mid \partial_{x}^{k} v(0)=0,0 \leq k \leq m-1\right\} .
$$

For any $v \in C_{0, m, \beta}^{m-1}(\Lambda)$ and $m \geq 1$, the auxiliary interpolation $\mathcal{I}_{N,-m, \beta} v$ $\in Q_{N}^{(-m, \beta)}(\Lambda)$ is determined uniquely by

$$
\mathcal{I}_{N,-m, \beta} v\left(\xi_{N, j}^{(m, \beta)}\right)=v\left(\xi_{N, j}^{(m, \beta)}\right), \quad 0 \leq j \leq N-m .
$$

The following lemma describes the stability of interpolation $\mathcal{I}_{N,-m, \beta} v$.

Lemma 5.1. For any $v \in C_{0, m, \beta}^{m-1}(\Lambda) \cap H_{\omega_{-m, \beta}, A}^{1}(\Lambda)$ and integers $1 \leq m \leq N$,

$$
\left\|\mathcal{I}_{N,-m, \beta} v\right\|_{\omega_{-m, \beta}} \leq c\left(\|v\|_{\omega_{-m, \beta}}+\beta^{-\frac{1}{2}}\left((\ln N)^{\frac{1}{2}}+N^{-\frac{1}{2}}\right)\left\|\partial_{x} v\right\|_{\omega_{-m+1, \beta}}\right) .
$$

Proof. Let $\aleph_{j}^{\prime}$ be the set of all $j$ such that $0<\xi_{N, j}^{(m, \beta)} \leq \frac{\eta}{\beta}$, while $\aleph_{j}^{\prime \prime}$ stands for the set of all $j$ such that $\xi_{N, j}^{(m, \beta)}>\frac{\eta}{\beta}$. By virtue of (5.9), we have

$$
\left\|\mathcal{I}_{N,-m, \beta} v\right\|_{\omega_{-m, \beta}}^{2}=\sum_{j=0}^{N-m} v^{2}\left(\xi_{N, j}^{(m, \beta)}\right) \omega_{N, j}^{(m, \beta)}=A_{N}+B_{N}
$$

where

$$
A_{N}=\sum_{j \in \aleph_{j}^{\prime}} v^{2}\left(\xi_{N, j}^{(m, \beta)}\right) \omega_{N, j}^{(m, \beta)}, \quad B_{N}=\sum_{j \in \aleph_{j}^{\prime \prime}} v^{2}\left(\xi_{N, j}^{(m, \beta)}\right) \omega_{N, j}^{(m, \beta)} .
$$

We first estimate $A_{N}$. For simplicity of statements, let

$$
\begin{array}{ll}
\Delta_{j}^{(m, \beta)}=\left[\xi_{N, j-1}^{(m, \beta)}, \xi_{N, j}^{(m, \beta)}\right], & \left|\Delta_{j}^{(m, \beta)}\right|=\xi_{N, \beta}^{(m, \beta)}-\xi_{N, j-1}^{(m, \beta)}, \\
\delta_{j,+}^{(m, \beta)}=\left(\xi_{N, j}^{(m, \beta)}\right)^{\frac{1}{2}}+\left(\xi_{N, j-1}^{(m, \beta)}\right)^{\frac{1}{2}}, & \delta_{j,-}^{(m, \beta)}=\left(\xi_{N, j}^{(m, \beta)}\right)^{\frac{1}{2}}-\left(\xi_{N, j-1}^{(m, \beta)}\right)^{\frac{1}{2}} .
\end{array}
$$


We use (3.9) and (5.7) to obtain

$$
\begin{aligned}
A_{N} & \leq \frac{c}{\sqrt{\beta(N-m)}} \sum_{j \in \aleph_{j}^{\prime}}\left(\xi_{N, j}^{(m, \beta)}\right)^{\frac{1}{2}} \sup _{x \in \Delta_{j}^{(m, \beta)}}\left|x^{-m} v^{2}\right| \\
& \leq \frac{c}{\sqrt{\beta(N-m)}} \sum_{j \in \aleph_{j}^{\prime}}\left(\left(\xi_{N, j}^{(m, \beta)}\right)^{\frac{1}{2}}\left(\delta_{j,+}^{(m, \beta)}\right)^{-1}\left(\delta_{j,-}^{(m, \beta)}\right)^{-1}\left\|x^{-\frac{m}{2}} v(x)\right\|_{L^{2}\left(\Delta_{j}^{(m, \beta)}\right)}^{2}\right. \\
& \left.+\left(\xi_{N, j}^{(m, \beta)}\right)^{\frac{1}{2}} \delta_{j,+}^{(m, \beta)} \delta_{j,-}^{(m, \beta)}\left(\left\|x^{-\frac{m}{2}} \partial_{x} v\right\|_{L^{2}\left(\Delta_{j}^{(m, \beta)}\right)}^{2}+\left\|x^{-\frac{m}{2}-1} v\right\|_{L^{2}\left(\Delta_{j}^{(m, \beta)}\right)}^{2}\right)\right) .
\end{aligned}
$$

We now estimate the upper-bound of the right side of the above inequality. By (5.4),

$$
\begin{aligned}
& \left(\xi_{N, j}^{(m, \beta)}\right)^{\frac{1}{2}} \delta_{j,+}^{(m, \beta)} \delta_{j,-}^{(m, \beta)}\left\|x^{-\frac{m}{2}-1} v\right\|_{L^{2}\left(\Delta_{j}^{(m, \beta)}\right)}^{2} \\
& \quad \leq\left(\xi_{N, j}^{(m, \beta)}\right)^{\frac{1}{2}}\left[\left(\xi_{N, j}^{(m, \beta)}\right)^{\frac{1}{2}}+\left(\xi_{N, j-1}^{(m, \beta)}\right)^{\frac{1}{2}}\right]^{2}\left\|x^{-\frac{m}{2}-1} v\right\|_{L^{2}\left(\Delta_{j}^{(m, \beta)}\right)}^{2} \\
& \quad \leq c\left(\xi_{N, j}^{(m, \beta)}\right)^{\frac{3}{2}}\left(\xi_{N, j-1}^{(m, \beta)}\right)^{-2}\left\|x^{-\frac{m}{2}} v\right\|_{L^{2}\left(\Delta_{j}^{(m, \beta)}\right)}^{2} \\
& \quad \leq c \sqrt{\beta(N-m)}\left\|x^{-\frac{m}{2}} v\right\|_{L^{2}\left(\Delta_{j}^{(m, \beta)}\right)}^{2}
\end{aligned}
$$

Similarly,

$$
\begin{aligned}
& \left(\xi_{N, j}^{(m, \beta)}\right)^{\frac{1}{2}} \delta_{j,+}^{(m, \beta)} \delta_{j,-}^{(m, \beta)}\left\|x^{-\frac{m}{2}} \partial_{x} v\right\|_{L^{2}\left(\Delta_{j}^{(m, \beta)}\right)}^{2} \\
& \quad \leq c\left(\xi_{N, j}^{(m, \beta)}\right)^{\frac{3}{2}}\left(\xi_{N, j-1}^{(m, \beta)}\right)^{-1}\left\|x^{-\frac{m}{2}+\frac{1}{2}} \partial_{x} v\right\|_{L^{2}\left(\Delta_{j}^{(m, \beta)}\right)}^{2} \\
& \quad \leq \frac{c}{\sqrt{\beta(N-m)}}\left\|x^{-\frac{m}{2}+\frac{1}{2}} \partial_{x} v\right\|_{L^{2}\left(\Delta_{j}^{(m, \beta)}\right)}^{2}
\end{aligned}
$$

Furthermore, we use (5.4) again to show that for $0<\xi_{N, j}^{(m, \beta)} \leq \frac{\eta}{\beta}$,

$$
\delta_{j,-}^{(m, \beta)} \sim \frac{1}{\sqrt{\beta(N-m)}}, \quad\left(\xi_{N, j}^{(m, \beta)}\right)^{\frac{1}{2}}\left(\delta_{j,+}^{(m, \beta)}\right)^{-1} \leq c .
$$

Consequently,

$$
\begin{aligned}
A_{N} & \leq c \sum_{j \in \aleph_{j}^{\prime}}\left(\left\|x^{-\frac{m}{2}} v\right\|_{L^{2}\left(\Delta_{j}^{(m, \beta)}\right)}^{2}+\beta^{-1} N^{-1}\left\|x^{-\frac{m}{2}+\frac{1}{2}} \partial_{x} v\right\|_{L^{2}\left(\Delta_{j}^{(m, \beta)}\right)}^{2}\right) \\
& \leq c\left(\|v\|_{\omega_{-m, \beta}}^{2}+\beta^{-1} N^{-1}\left\|\partial_{x} v\right\|_{\omega_{-m+1, \beta}}^{2}\right) .
\end{aligned}
$$

Next, we estimate $B_{N}$. Since $v \in L_{\omega_{-m, \beta}}^{2}(\Lambda)$, we have that $v^{2}(x) x^{-m+1} e^{-\beta x} \rightarrow 0$ as $x \rightarrow 0$. Thus,

$$
v^{2}(x) x^{-m+1} e^{-\beta x}=\int_{0}^{x} \partial_{\xi}\left(v^{2}(\xi) \xi^{-m+1} e^{-\beta \xi}\right) d \xi .
$$

A direct calculation shows

$$
\begin{aligned}
v^{2}(x) x^{-m+1} e^{-\beta x} & +(m-1) \int_{0}^{x} v^{2}(\xi) \xi^{-m} e^{-\beta \xi} d \xi+\beta \int_{0}^{x} v^{2}(\xi) \xi^{-m+1} e^{-\beta \xi} d \xi \\
& =2 \int_{0}^{x} v(\xi) \partial_{\xi} v(\xi) \xi^{-m+1} e^{-\beta \xi} d \xi .
\end{aligned}
$$

Thanks to $m \geq 1$, we obtain

$$
v^{2}(x) x^{-m+1} e^{-\beta x} \leq \frac{1}{\beta}\left\|\partial_{x} v\right\|_{\omega_{-m+1, \beta}}^{2} .
$$


Therefore, with the aid of (5.6), (5.8) and (5.13), we deduce that

$$
\begin{aligned}
B_{N} & \leq c \sum_{j \in \aleph_{j}^{\prime \prime}} v^{2}\left(\xi_{N, j}^{(m, \beta)}\right) \omega_{-m, \beta}\left(\xi_{N, j}^{(m, \beta)}\right)\left(\xi_{N, j}^{(m, \beta)}-\xi_{N, j-1}^{(m, \beta)}\right) \\
& \leq c \sup _{x>\frac{\eta}{\beta}}\left|v^{2}(x) \omega_{-m+1, \beta}(x)\right| \sum_{j \in \aleph_{j}^{\prime \prime}} \frac{1}{\xi_{N, j}^{(m, \beta)}}\left(\xi_{N, j}^{(m, \beta)}-\xi_{N, j-1}^{(m, \beta)}\right) \\
& \leq c \sup _{x>\frac{\eta}{\beta}}\left|v^{2}(x) \omega_{-m+1, \beta}(x)\right| \int_{\eta \beta^{-1}}^{4 \beta^{-1}(N-m+1)} \frac{1}{x} d x \\
& \leq \frac{c}{\beta} \ln N\left\|\partial_{x} v\right\|_{\omega_{-m+1, \beta}}^{2} .
\end{aligned}
$$

Finally, the desired result follows from a combination of (5.11), (5.12) and (5.14).

We are now in position to estimate the error of interpolation $\mathcal{I}_{N,-m, \beta} v$.

Lemma 5.2. If $v \in{ }_{0} H_{\omega_{-m, \beta}, A}^{m}(\Lambda), \partial_{x}^{r} v \in L_{\omega_{-m+r, \beta}}^{2}(\Lambda)$, integers $1 \leq m \leq \min (r, N)$ and $0 \leq k \leq r \leq N+1$, then

$$
\begin{aligned}
& \left\|\partial_{x}^{k}\left(\mathcal{I}_{N,-m, \beta} v-v\right)\right\|_{\omega_{-m+k, \beta}} \\
& \quad \leq c\left(\beta^{-\frac{1}{2}}+1\right)(\ln N)^{\frac{1}{2}}(\beta N)^{\frac{k+1-r}{2}}\left\|\partial_{x}^{r} v\right\|_{\omega_{-m+r, \beta} .}
\end{aligned}
$$

Proof. Clearly, $\mathcal{I}_{N,-m, \beta} v$ is meaningful. Since $\mathcal{I}_{N,-m, \beta} P_{N,-m, \beta} v=P_{N,-m, \beta} v$, we use $(4.30),(5.10)$ and (4.33) successively to deduce that

$$
\begin{aligned}
\| \partial_{x}^{k}\left(P_{N,-m, \beta} v-\right. & \left.\mathcal{I}_{N,-m, \beta} v\right)\left\|_{\omega_{-m+k, \beta}} \leq c(\beta N)^{\frac{k}{2}}\right\| \mathcal{I}_{N,-m, \beta}\left(P_{N,-m, \beta} v-v\right) \|_{\omega_{-m, \beta}} \\
\leq & c(\beta N)^{\frac{k}{2}}\left(\left\|P_{N,-m, \beta} v-v\right\|_{\omega_{-m, \beta}}\right. \\
& \left.+\beta^{-\frac{1}{2}}\left((\ln N)^{\frac{1}{2}}+N^{-\frac{1}{2}}\right)\left\|\partial_{x}\left(P_{N,-m, \beta} v-v\right)\right\|_{\omega_{-m+1, \beta}}\right) \\
\leq & c\left(\beta^{-\frac{1}{2}}+1\right)(\ln N)^{\frac{1}{2}}(\beta N)^{\frac{k+1-r}{2}}\left\|\partial_{x}^{r} v\right\|_{\omega_{-m+r, \beta} .}
\end{aligned}
$$

Finally, we use (4.33) again to obtain

$$
\begin{aligned}
\left\|\partial_{x}^{k}\left(\mathcal{I}_{N,-m, \beta} v-v\right)\right\|_{\omega_{-m+k, \beta} \leq} \leq & \left\|\partial_{x}^{k}\left(P_{N,-m, \beta} v-v\right)\right\|_{\omega_{-m+k, \beta}} \\
& +\left\|\partial_{x}^{k}\left(P_{N,-m, \beta} v-\mathcal{I}_{N,-m, \beta} v\right)\right\|_{\omega_{-m+k, \beta}} \\
\leq & c\left(\beta^{-\frac{1}{2}}+1\right)(\ln N)^{\frac{1}{2}}(\beta N)^{\frac{k+1-r}{2}}\left\|\partial_{x}^{r} v\right\|_{\omega_{-m+r, \beta}} .
\end{aligned}
$$

This ends the proof.

We are now in position to consider the generalized Laguerre-Gauss-Radau interpolation for functions with nonhomogenous boundary values. Let $v_{b, m}(x)$ be the same as in (4.36). For any $v \in C_{m, \beta}^{m-1}(\Lambda)$, we set $\tilde{v}(x)=v(x)-v_{b, m}(x)$, Then $\tilde{v} \in C_{0, m, \beta}^{m-1}(\Lambda)$. Thus, there exists the interpolation $\mathcal{I}_{N,-m, \beta} \tilde{v} \in Q_{N}^{(-m, \beta)}(\Lambda)$. Further, we define the new interpolation as

$$
\mathcal{I}_{R, N,-m, \beta} v(x)=\mathcal{I}_{N,-m, \beta} \tilde{v}(x)+v_{b, m}(x) .
$$

It can be checked that

$$
\begin{array}{ll}
\mathcal{I}_{R, N,-m, \beta} v\left(\xi_{N, j}^{(m, \beta)}\right)=v\left(\xi_{N, j}^{(m, \beta)}\right), & 0 \leq j \leq N-m, \\
\mathcal{I}_{R, N,-m, \beta} v(0)=\partial_{x}^{k} v(0), & 0 \leq k \leq m-1 .
\end{array}
$$


This interpolation is the same as the generalized Laguerre-Gauss-Radau interpolation, since both of them are polynomials of degree $N$, with the same interpolation nodes, and satisfy the same condition (5.16).

Next, we estimate the error of interpolation $\mathcal{I}_{R, N,-m, \beta} v$. Clearly, $\mathcal{I}_{R, N,-m, \beta} v(x)-$ $v(x)=\mathcal{I}_{N,-m, \beta} \tilde{v}(x)-\tilde{v}(x)$. This fact, along with (5.15), leads to the following conclusion.

Theorem 5.1. If $v \in H_{\omega_{-m, \beta}, A}^{m}(\Lambda), \partial_{x}^{r} v \in L_{\omega_{-m+r, \beta}}^{2}(\Lambda)$, integers $1 \leq m \leq \min (r, N)$ and $0 \leq k \leq r \leq N+1$, then

$$
\left\|\partial_{x}^{k}\left(\mathcal{I}_{R, N,-m, \beta} v-v\right)\right\|_{\omega_{-m+k, \beta}} \leq c\left(\beta^{-\frac{1}{2}}+1\right)(\ln N)^{\frac{1}{2}}(\beta N)^{\frac{k+1-r}{2}}\left\|\partial_{x}^{r} v\right\|_{\omega_{-m+r, \beta}} .
$$

Remark 5.1. The result (5.17) improves the existing results essentially. For instance, (5.17) with $m=1$ implies

$$
\left\|\partial_{x}^{k}\left(\mathcal{I}_{R, N,-1, \beta} v-v\right)\right\|_{\omega_{-1+k, \beta}} \leq c\left(\beta^{-\frac{1}{2}}+1\right)(\ln N)^{\frac{1}{2}}(\beta N)^{\frac{k+1-r}{2}}\left\|\partial_{x}^{r} v\right\|_{\omega_{-1+r, \beta}} .
$$

However, it was only shown before that (cf. (3.33) of [22]) for $\alpha>-1,1 \leq k \leq r \leq$ $N+1$ and $r>\alpha+1($ or $|\alpha|<1)$,

$$
\begin{aligned}
& \left\|\partial_{x}^{k}\left(\mathcal{I}_{R, N, \alpha, \beta} v-v\right)\right\|_{\omega_{\alpha, \beta}} \leq c(\beta N)^{\frac{2 k+1-r}{2}}\left(\beta^{-1}\left\|\partial_{x}^{r} v\right\|_{\omega_{-1+r+\alpha, \beta}}\right. \\
& \left.\quad+\beta^{-1} N^{-\frac{1}{2}}\left\|\partial_{x}^{r} v\right\|_{\omega_{-k+r+\alpha, \beta}}+\left(1+\beta^{-\frac{1}{2}}\right)(\ln N)^{\frac{1}{2}}\left\|\partial_{x}^{r} v\right\|_{\omega_{r+\alpha, \beta}}\right) .
\end{aligned}
$$

Finally, if $v \in H_{\omega_{-m, \beta}, A}^{m}(\Lambda), \partial_{x}^{r} v \in L_{\omega_{-m+r, \beta}}^{2}(\Lambda)$, integers $1 \leq m \leq \min (r, N)$ and $r \leq N+1$, then for any $\phi \in Q_{N}^{(-m, \beta)}(\Lambda)$,

$$
\begin{aligned}
& \left|(v, \phi)_{\omega_{-m, \beta}}-(v, \phi)_{N, m, \beta}\right| \\
& \quad \leq c\left(\beta^{-\frac{1}{2}}+1\right)(\ln N)^{\frac{1}{2}}(\beta N)^{\frac{1-r}{2}}\left\|\partial_{x}^{r} v\right\|_{\omega_{-m+r, \beta}}\|\phi\|_{\omega_{-m, \beta}} .
\end{aligned}
$$

\section{APPENDIX}

In this appendix, we prove the estimate (2.24). It suffices to verify that for $1 \leq m, n \leq 4$ and $\max (m, n) \leq r \leq m+n-1$,

$$
\left\|\partial_{x}^{r} v_{m, n, b}\right\|_{\chi^{(-m+r,-n+r)}} \leq c\left\|\partial_{x}^{r} v\right\|_{\chi^{(-m+r,-n+r)}} .
$$

We need some preparations. First, for any function $w$,

$$
b_{0}(w):=-w(-1)+w(1)=\int_{\Lambda} \partial_{x} w(x) d x .
$$

Next, by virtue of (A.2) and integration by parts, we get

$$
\begin{aligned}
b_{1}(w) & :=w(-1)-w(1)+\partial_{x} w(-1)+\partial_{x} w(1)=\int_{\Lambda} x \partial_{x}^{2} w(x) d x \\
& =\frac{1}{2} \int_{\Lambda}\left(1-x^{2}\right) \partial_{x}^{3} w(x) d x .
\end{aligned}
$$

Furthermore, with the aid of (A.3), (A.2) and integration by parts, we obtain that

$$
\begin{aligned}
b_{2}(w) & :=3\left(w(-1)-w(1)+\partial_{x} w(-1)+\partial_{x} w(1)\right)+\partial_{x}^{2} w(-1)-\partial_{x}^{2} w(1) \\
& =3 b_{1}(w)-b_{0}\left(\partial_{x}^{2} w\right)=\frac{3}{2} \int_{\Lambda}\left(1-x^{2}\right) \partial_{x}^{3} w(x) d x-\int_{\Lambda} \partial_{x}^{3} w(x) d x \\
& =\frac{1}{2} \int_{\Lambda}\left(1-3 x^{2}\right) \partial_{x}^{3} w(x) d x=-\frac{1}{2} \int_{\Lambda} x\left(1-x^{2}\right) \partial_{x}^{4} w(x) d x \\
& =-\frac{1}{8} \int_{\Lambda}\left(1-x^{2}\right)^{2} \partial_{x}^{5} w(x) d x .
\end{aligned}
$$


Finally, using (A.4), (A.3) and integration by parts, we deduce that

$$
\begin{aligned}
b_{3}(w):= & 15\left(w(-1)-w(1)+\partial_{x} w(-1)+\partial_{x} w(1)\right) \\
& +6\left(\partial_{x}^{2} w(-1)-\partial_{x}^{2} w(1)\right)+\partial_{x}^{3} w(-1)+\partial_{x}^{3} w(1) \\
= & 5 b_{2}(w)+b_{1}\left(\partial_{x}^{2} w\right)=-\frac{5}{8} \int_{\Lambda}\left(1-x^{2}\right)^{2} \partial_{x}^{5} w(x) d x \\
& +\frac{1}{2} \int_{\Lambda}\left(1-x^{2}\right) \partial_{x}^{5} w(x) d x \\
= & \frac{1}{8} \int_{\Lambda}\left(-1+6 x^{2}-5 x^{4}\right) \partial_{x}^{5} w(x) d x \\
= & \frac{1}{8} \int_{\Lambda} x\left(1-x^{2}\right)^{2} \partial_{x}^{6} w(x) d x=\frac{1}{48} \int_{\Lambda}\left(1-x^{2}\right)^{3} \partial_{x}^{7} w(x) d x .
\end{aligned}
$$

We now consider (A.1) with $m=n=1$. By (2.17), we have (also see [27])

$$
v_{1,1, b}(x)=\frac{1}{2}(v(-1)(1-x)+v(1)(1+x)) .
$$

Obviously, $\partial_{x} v_{1,1, b}(x)=\frac{1}{2} b_{0}(v)$. Thus, we use (A.2) and the Cauchy inequality to obtain

$$
\left\|\partial_{x} v_{1,1, b}\right\| \leq\left|b_{0}(v)\right| \leq c\left\|\partial_{x} v\right\| .
$$

This is the desired result (A.1) with $m=n=1$ and $r=1$.

Next, we consider the case with $m=n=2$. By (2.17), we have

$$
\begin{aligned}
v_{2,2, b}(x)= & \frac{1}{4}\left(v(-1)\left(x^{3}-3 x+2\right)+v(1)\left(-x^{3}+3 x+2\right)\right. \\
& \left.+\partial_{x} v(-1)\left(x^{3}-x^{2}-x+1\right)+\partial_{x} v(1)\left(x^{3}+x^{2}-x-1\right)\right) .
\end{aligned}
$$

A calculation shows $\partial_{x}^{3} v_{2,2, b}(x)=\frac{3}{2} b_{1}(v)$. Consequently, we use (A.3) to obtain

$$
\left\|\partial_{x}^{3} v_{2,2, b}\right\|_{\chi^{(1,1)}} \leq c\left|b_{1}(v)\right| \leq c|| \partial_{x}^{3} v \|_{\chi^{(1,1)}}
$$

This is the result (A.1) with $m=n=2$ and $r=3$. Furthermore,

$$
\partial_{x}^{2} v_{2,2, b}(x)=\frac{3}{2} x b_{1}(v)+\frac{1}{2} b_{0}\left(\partial_{x} v\right) .
$$

Thereby, we use the first equality of (A.3) and (A.2) to obtain

$$
|| \partial_{x}^{2} v_{2,2, b}\left\|\leq 3\left|b_{1}(v)\right|+\left|b_{0}\left(\partial_{x} v\right)\right| \leq c|| \partial_{x}^{2} v\right\| \mid .
$$

This is the result (A.1) with $m=n=2$ and $r=2$.

Third, we deal with the case with $m=n=3$. A calculation shows

$$
\begin{aligned}
v_{3,3, b}(x)= & \frac{1}{16}\left(v(-1)\left(-3 x^{5}+10 x^{3}-15 x+8\right)+v(1)\left(3 x^{5}-10 x^{3}+15 x+8\right)\right. \\
& +\partial_{x} v(-1)\left(-3 x^{5}+x^{4}+10 x^{3}-6 x^{2}-7 x+5\right) \\
& +\partial_{x} v(1)\left(-3 x^{5}-x^{4}+10 x^{3}+6 x^{2}-7 x-5\right) \\
& +\partial_{x}^{2} v(-1)\left(-x^{5}+x^{4}+2 x^{3}-2 x^{2}-x+1\right) \\
& \left.+\partial_{x}^{2} v(1)\left(x^{5}+x^{4}-2 x^{3}-2 x^{2}+x+1\right)\right) .
\end{aligned}
$$

It can be checked that $\partial_{x}^{5} v_{3,3, b}(x)=-\frac{15}{2} b_{2}(v)$. Then, an argument as in the derivation of (A.6), along with (A.4), leads to $\left\|\partial_{x}^{5} v_{3,3, b}\right\|_{\chi^{(2,2)}} \leq c\left\|\partial_{x}^{5} v\right\|_{\chi^{(2,2)}}$. Moreover,

$$
\partial_{x}^{4} v_{3,3, b}(x)=-\frac{15}{2} x b_{2}(v)+\frac{3}{2} b_{1}\left(\partial_{x} v\right) .
$$


Thereby, we use the fourth equality of (A.4) and (A.3) to obtain $\left\|\partial_{x}^{4} v_{3,3, b}\right\|_{\chi^{(1,1)}} \leq$ $c\left\|\partial_{x}^{4} v\right\|_{\chi^{(1,1)}}$. Furthermore,

$$
\partial_{x}^{3} v_{3,3, b}(x)=-\frac{15}{4} x^{2} b_{2}(v)+\frac{3}{2} x b_{1}\left(\partial_{x} v\right)+\frac{3}{4} b_{2}(v)+\frac{3}{2} b_{1}(v) .
$$

We can use the second equality of (A.4) and the first two equalities of (A.3) to estimate the upper-bounds of $\left|b_{2}(v)\right|,\left|b_{1}\left(\partial_{x} v\right)\right|$ and $\left|b_{1}(v)\right|$, respectively. Finally, $\left\|\partial_{x}^{3} v_{3,3, b}\right\| \leq c\left\|\partial_{x}^{3} v\right\|$. The previous three estimates imply (A.1) with $m=n=3$ and $r=5,4,3$.

We now turn to the case with $m=n=4$. We have

$$
\begin{aligned}
v_{4,4, b}(x)= & \frac{1}{32}\left(v(-1)\left(5 x^{7}-21 x^{5}+35 x^{3}-35 x+16\right)\right. \\
& +v(1)\left(-5 x^{7}+21 x^{5}-35 x^{3}+35 x+16\right) \\
& +\partial_{x} v(-1)\left(5 x^{7}-x^{6}-21 x^{5}+5 x^{4}+35 x^{3}-15 x^{2}-19 x+11\right) \\
& +\partial_{x} v(1)\left(5 x^{7}+x^{6}-21 x^{5}-5 x^{4}+35 x^{3}+15 x^{2}-19 x-11\right) \\
& +\partial_{x}^{2} v(-1)\left(2 x^{7}-x^{6}-8 x^{5}+5 x^{4}+10 x^{3}-7 x^{2}-4 x+3\right) \\
& \left.+\partial_{x}^{2} v(1)\left(-2 x^{7}-x^{6}+8 x^{5}+5 x^{4}-10 x^{3}-7 x^{2}+4 x+3\right)\right) \\
& +\frac{1}{96}\left(\partial_{x}^{3} v(-1)\left(x^{7}-x^{6}-3 x^{5}+3 x^{4}+3 x^{3}-3 x^{2}-x+1\right)\right. \\
& \left.+\partial_{x}^{3} v(1)\left(x^{7}+x^{6}-3 x^{5}-3 x^{4}+3 x^{3}+3 x^{2}-x-1\right)\right) .
\end{aligned}
$$

It can be checked that $\partial_{x}^{7} v_{4,4, b}(x)=\frac{105}{2} b_{3}(v)$. This with (A.5) leads to $\left\|\partial_{x}^{7} v_{4,4, b}\right\|_{\chi^{(3,3)}}$ $\leq c\left\|\partial_{x}^{7} v\right\|_{\chi^{(3,3)}}$. Next, $\partial_{x}^{6} v_{4,4, b}(x)=\frac{15}{2}\left(7 x b_{3}(v)-b_{2}\left(\partial_{x} v\right)\right)$. Hence, we use the fifth equality of (A.5) and (A.4) to verify that $\left\|\partial_{x}^{6} v_{4,4, b}\right\|_{\chi^{(2,2)}} \leq c\left\|\partial_{x}^{6} v\right\|_{\chi^{(2,2)}}$. Furthermore,

$$
\partial_{x}^{5} v_{4,4, b}(x)=\frac{15}{4}\left(7 x^{2} b_{3}(v)-2 x b_{2}\left(\partial_{x} v\right)-7 b_{2}(v)-b_{1}\left(\partial_{x}^{2} v\right)\right) .
$$

Thus, we could use the third equality of (A.5), the last two equalities of (A.4) and (A.3) to estimate the upper-bounds of the terms $\left|b_{3}(v)\right|,\left|b_{2}\left(\partial_{x} v\right)\right|,\left|b_{2}(v)\right|$ and $\left|b_{1}\left(\partial_{x}^{2} v\right)\right|$, respectively. As a result, we obtain $\left\|\partial_{x}^{5} v_{4,4, b}\right\|_{\chi^{(1,1)}} \leq c\left\|\partial_{x}^{5} v\right\|_{\chi^{(1,1)}}$. Furthermore,

$\partial_{x}^{4} v_{4,4, b}(x)=\frac{35}{4} x^{3} b_{3}(v)-\frac{15}{4} x^{2} b_{2}\left(\partial_{x} v\right)-\frac{15}{4} x b_{3}(v)-\frac{15}{2} x b_{2}(v)+\frac{3}{4} b_{2}\left(\partial_{x} v\right)+\frac{3}{2} b_{1}\left(\partial_{x} v\right)$.

We can estimate $\left|b_{2}\left(\partial_{x} v\right)\right|,\left|b_{2}(v)\right|$ and $\left|b_{1}\left(\partial_{x} v\right)\right|$, by using the fourth equality of (A.4), the fifth equality of (A.4) and (A.3), respectively. On the other hand, according to (A.5), the fifth equality of (A.4) and the first equality of (A.3), we have

$$
\begin{aligned}
\left|b_{3}(v)\right| & =\left|5 b_{2}(v)+b_{1}\left(\partial_{x}^{2} v\right)\right|=\left|-\frac{5}{2} \int_{\Lambda} x\left(1-x^{2}\right) \partial_{x}^{4} v(x) d x+\int_{\Lambda} x \partial_{x}^{4} v(x) d x\right| \\
& \leq c\left\|\partial_{x}^{4} v\right\| .
\end{aligned}
$$

Finally, we conclude that $\left\|\partial_{x}^{4} v_{4,4, b}\right\| \leq c\left\|\partial_{x}^{4} v\right\|$. The previous statements imply (A.1) with $m=n=4$ and $r=7,6,5,4$.

We now consider the cases with $m \neq n$. First, let $m=1$ and $n=2$. We have

$$
v_{1,2, b}(x)=\frac{1}{4}\left(v(-1)\left(-x^{2}-2 x+3\right)+v(1)\left(x^{2}+2 x+1\right)+2 \partial_{x} v(-1)\left(1-x^{2}\right)\right) .
$$


A calculation, along with (A.2) and the second equality of (A.3), shows that

$$
\partial_{x}^{2} v_{1,2, b}(x)=-\frac{1}{2}\left(b_{1}(v)-b_{0}\left(\partial_{x} v\right)\right)=\frac{1}{2} \int_{\Lambda}(1-x) \partial_{x}^{2} v(x) d x
$$

Accordingly, $\left\|\partial_{x}^{2} v_{1,2, b}\right\|_{\chi^{(1,0)}} \leq c\left\|\partial_{x}^{2} v\right\|_{\chi^{(1,0)}}$. This is (A.1) with $m=1, n=2$ and $r=2$. In the same manner, we verify that $\left\|\partial_{x}^{2} v_{2,1, b}\right\|_{\chi^{(0,1)}} \leq c\left\|\partial_{x}^{2} v\right\|_{\chi^{(0,1)}}$, which is (A.1) with $m=2, n=1$ and $r=2$.

Next, we consider the case with $m=1$ and $n=3$. We have

$$
\begin{aligned}
v_{1,3, b}(x)= & \frac{1}{8}\left(v(-1)\left(-x^{3}-3 x^{2}-3 x+7\right)+v(1)\left(x^{3}+3 x^{2}+3 x+1\right)\right) \\
& +\frac{1}{4}\left(\partial_{x} v(-1)\left(-x^{3}-3 x^{2}+x+3\right)+\partial_{x}^{2} v(-1)\left(-x^{3}-x^{2}+x+1\right)\right) .
\end{aligned}
$$

A calculation with the last two equalities of (A.3) and (A.2), yields

$$
\partial_{x}^{3} v_{1,3, b}(x)=-\frac{3}{4}\left(b_{1}(v)+b_{1}\left(\partial_{x} v\right)-b_{0}\left(\partial_{x}^{2} v\right)\right)=\frac{3}{8} \int_{\Lambda}(1-x)^{2} \partial_{x}^{3} v(x) d x .
$$

This leads to $\left\|\partial_{x}^{3} v_{1,3, b}\right\|_{\chi^{(2,0)}} \leq c\left\|\partial_{x}^{3} v\right\|_{\chi^{(2,0)}}$. Similarly, $\left\|\partial_{x}^{3} v_{3,1, b}\right\|_{\chi^{(0,2)}} \leq c\left\|\partial_{x}^{3} v\right\|_{\chi^{(0,2)}}$ for $m=3$ and $n=1$. The above two inequalities imply the validity of (A.1) for $m=1, n=3$ and $m=3, n=1$.

Third, we consider the case with $m=1$ and $n=4$. We have

$$
\begin{aligned}
& v_{1,4, b}(x)=\frac{1}{16}\left(v(-1)\left(-x^{4}-4 x^{3}-6 x^{2}-4 x+15\right)+v(1)\left(x^{4}+4 x^{3}+6 x^{2}+4 x+1\right)\right) \\
& +\frac{1}{8}\left(\partial_{x} v(-1)\left(-x^{4}-4 x^{3}-6 x^{2}+4 x+7\right)+\partial_{x}^{2} v(-1)\left(-x^{4}-4 x^{3}-2 x^{2}+4 x+3\right)\right) \\
& +\frac{1}{12} \partial_{x}^{3} v(-1)\left(-x^{4}-2 x^{3}+2 x+1\right) .
\end{aligned}
$$

A calculation, along with the fifth equality of (A.4), the last two equalities of (A.3) and (A.2), gives

$$
\partial_{x}^{4} v_{1,4, b}(x)=-\frac{1}{2}\left(b_{2}(v)+3 b_{1}\left(\partial_{x} v\right)+b_{1}\left(\partial_{x}^{2} v\right)-2 b_{0}\left(\partial_{x}^{3} v\right)\right)=\frac{1}{4} \int_{\Lambda}(1-x)^{3} \partial_{x}^{4} v(x) d x .
$$

Therefore, $\left\|\partial_{x}^{4} v_{1,4, b}\right\|_{\chi^{(3,0)}} \leq c\left\|\partial_{x}^{4} v\right\|_{\chi^{(3,0)}}$. Similarly, $\left\|\partial_{x}^{4} v_{4,1, b}\right\|\left\|_{\chi^{(0,3)}} \leq c\right\| \partial_{x}^{4} v \|_{\chi^{(0,3)}}$ for $m=4$ and $n=1$.

Further, we deal with the case with $m=2$ and $n=3$. A direct calculation yields

$$
\begin{aligned}
v_{2,3, b}(x)= & \frac{1}{16}\left(v(-1)\left(3 x^{4}+4 x^{3}-6 x^{2}-12 x+11\right)\right. \\
& \left.+v(1)\left(-3 x^{4}-4 x^{3}+6 x^{2}+12 x+5\right)\right) \\
& +\frac{1}{4} \partial_{x} v(-1)\left(x^{4}+x^{3}-3 x^{2}-x+2\right)+\frac{1}{8} \partial_{x} v(1)\left(x^{4}+2 x^{3}-2 x-1\right) \\
& +\frac{1}{8} \partial_{x}^{2} v(-1)\left(x^{4}-2 x^{2}+1\right) .
\end{aligned}
$$

Using the fifth equality of (A.4) and the last equality of (A.3), we verify that

$$
\partial_{x}^{4} v_{2,3, b}(x)=\frac{3}{2}\left(b_{2}(v)+b_{1}\left(\partial_{x} v\right)\right)=\frac{1}{2} \int_{\Lambda}(1+x)(1-x)^{2} \partial_{x}^{4} v(x) d x .
$$


Thus, $\left\|\partial_{x}^{4} v_{2,3, b}\right\|_{\chi^{(2,1)}} \leq c\left\|\partial_{x}^{4} v\right\|_{\chi^{(2,1)}}$. On the other hand, with the aid of the fourth equality of (A.4) and the last two equalities of (A.3), we obtain

$$
\begin{aligned}
\partial_{x}^{3} v_{2,3, b}(x) & =\frac{3}{2}\left(\left(b_{2}(v)+b_{1}\left(\partial_{x} v\right)\right) x+b_{1}(v)\right) \\
& =\frac{3}{4} x \int_{\Lambda}(1-x)(1+3 x) \partial_{x}^{3} v(x) d x+\frac{3}{4} \int_{\Lambda}\left(1-x^{2}\right) \partial_{x}^{3} v(x) d x
\end{aligned}
$$

This leads to $\left\|\partial_{x}^{3} v_{2,3, b}\right\|_{\chi^{(1,0)}} \leq c\left\|\partial_{x}^{3} v\right\|_{\chi^{(1,0)}}$. The above two results imply (A.1) with $m=2, n=3$ and $r=4,3$. Similarly, $\left\|\partial_{x}^{r} v_{3,2, b}\right\|_{\chi^{(-3+r,-2+r)}} \leq c\left\|\partial_{x}^{r} v\right\|_{\chi^{(-3+r,-2+r)}}$ for $m=3, n=2$ and $r=4,3$.

We now turn to the case with $m=2$ and $n=4$. A direct calculation yields

$$
\begin{aligned}
v_{2,4, b}(x) & =\frac{1}{16}\left(v(-1)\left(2 x^{5}+5 x^{4}-10 x^{2}-10 x+13\right)\right. \\
& +v(1)\left(-2 x^{5}-5 x^{4}+10 x^{2}+10 x+3\right) \\
& +\partial_{x} v(-1)\left(3 x^{5}+7 x^{4}-2 x^{3}-18 x^{2}-x+11\right) \\
& \left.+\partial_{x} v(1)\left(x^{5}+3 x^{4}+2 x^{3}-2 x^{2}-3 x-1\right)\right) \\
& +\frac{1}{8} \partial_{x}^{2} v(-1)\left(x^{5}+2 x^{4}-2 x^{3}-4 x^{2}+x+2\right) \\
& +\frac{1}{24} \partial_{x}^{3} v(-1)\left(x^{5}+x^{4}-2 x^{3}-2 x^{2}+x+1\right) .
\end{aligned}
$$

By using the last two equalities of (A.4) and (A.3), we derive that

$$
\partial_{x}^{5} v_{2,4, b}(x)=\frac{5}{2}\left(2 b_{2}(v)+b_{2}\left(\partial_{x} v\right)+b_{1}\left(\partial_{x}^{2} v\right)\right)=\frac{5}{8} \int_{\Lambda}(1+x)(1-x)^{3} \partial_{x}^{5} v(x) d x,
$$

which implies $\left\|\partial_{x}^{5} v_{2,4, b}\right\|_{\chi^{(3,1)}} \leq c\left\|\partial_{x}^{5} v\right\|_{\chi^{(3,1)}}$. Also, we have

$$
\partial_{x}^{4} v_{2,4, b}(x)=\frac{5}{2}\left(2 b_{2}(v)+b_{2}\left(\partial_{x} v\right)+b_{1}\left(\partial_{x}^{2} v\right)\right) x+\frac{1}{2}\left(b_{3}(v)+2 b_{2}\left(\partial_{x} v\right)+b_{0}\left(\partial_{x}^{3} v\right)\right) .
$$

Moreover, by virtue of the fourth and fifth equalities of (A.4) and the second equality of (A.3), we observe that

$$
2 b_{2}(v)+b_{2}\left(\partial_{x} v\right)+b_{1}\left(\partial_{x}^{2} v\right)=\frac{1}{2} \int_{\Lambda}(1-x)^{2}(1+2 x) \partial_{x}^{4} v(x) d x .
$$

On the other hand, with the aid of the fourth equality of (A.5), the fourth equality of (A.4) and integration by parts, we deduce that

$$
b_{3}(v)+2 b_{2}\left(\partial_{x} v\right)+b_{0}\left(\partial_{x}^{3} v\right)=\frac{1}{2} \int_{\Lambda}(1-x)^{2}(4+5 x) \partial_{x}^{4} v(x) d x .
$$

Thus, $\left\|\partial_{x}^{4} v_{2,4, b}\right\|_{\chi^{(2,0)}} \leq c\left\|\partial_{x}^{4} v\right\|_{\chi^{(2,0)}}$. The above two results imply (A.4) with $m=$ $2, n=4$ and $r=5,4$. Similarly, $\left\|\partial_{x}^{r} v_{4,2, b}\right\|_{\chi^{(-4+r,-2+r)}} \leq c\left\|\partial_{x}^{r} v\right\|_{\chi^{(-4+r,-2+r)}}$ for $m=4, n=2$ and $r=5,4$. 
Finally, we deal with the case with $m=3$ and $n=4$. A direct calculation yields

$$
\begin{aligned}
v_{3,4, b}(x)= & \frac{1}{32}\left(v(-1)\left(-5 x^{6}-6 x^{5}+15 x^{4}+20 x^{3}-15 x^{2}-30 x+21\right)\right. \\
& \left.+v(1)\left(5 x^{6}+6 x^{5}-15 x^{4}-20 x^{3}+15 x^{2}+30 x+11\right)\right) \\
& +\frac{1}{16}\left(\partial_{x} v(-1)\left(-3 x^{6}-3 x^{5}+10 x^{4}+10 x^{3}-15 x^{2}-7 x+8\right)\right. \\
& \left.+\partial_{x} v(1)\left(-2 x^{6}-3 x^{5}+5 x^{4}+10 x^{3}-7 x-3\right)\right) \\
& +\frac{1}{32}\left(\partial_{x}^{2} v(-1)\left(-3 x^{6}-2 x^{5}+11 x^{4}+4 x^{3}-13 x^{2}-2 x+5\right)\right. \\
& \left.+\partial_{x}^{2} v(1)\left(x^{6}+2 x^{5}-x^{4}-4 x^{3}-x^{2}+2 x+1\right)\right) \\
& +\frac{1}{48} \partial_{x}^{3} v(-1)\left(-x^{6}+3 x^{4}-3 x^{2}+1\right)
\end{aligned}
$$

This, along with the fifth equality of (A.5) and the last equality of (A.4), leads to

$$
\partial_{x}^{6} v_{3,4, b}(x)=-\frac{15}{2}\left(b_{3}(v)+b_{2}\left(\partial_{x} v\right)\right)=\frac{15}{16} \int_{\Lambda}(1+x)^{2}(1-x)^{3} \partial_{x}^{6} v(x) d x .
$$

Therefore, $\left\|\partial_{x}^{6} v_{3,4, b}\right\|_{\chi^{(3,2)}} \leq c\left\|\partial_{x}^{6} v\right\|_{\chi^{(3,2)}}$. Next, we use the fourth equality of (A.5) and the last two equalities of (A.4) to obtain

$$
\begin{aligned}
\partial_{x}^{5} v_{3,4, b}(x) & =-\frac{15}{2}\left(b_{3}(v)+b_{2}\left(\partial_{x} v\right)\right) x-\frac{15}{2} b_{2}(v) \\
& =\frac{15}{16} x \int_{\Lambda}(1-x)^{2}(1+x)(1+5 x) \partial_{x}^{5} v(x) d x+\frac{15}{16} \int_{\Lambda}\left(1-x^{2}\right)^{2} \partial_{x}^{5} v(x) d x
\end{aligned}
$$

Thereby, $\left\|\partial_{x}^{5} v_{3,4, b}\right\|_{\chi^{(2,1)}} \leq c\left\|\partial_{x}^{5} v\right\|_{\chi^{(2,1)}}$. Furthermore,

$\partial_{x}^{4} v_{3,4, b}(x)=-\frac{15}{4}\left(b_{3}(v)+b_{2}\left(\partial_{x} v\right)\right) x^{2}-\frac{15}{2} b_{2}(v) x+\frac{3}{4}\left(b_{3}(v)+b_{2}\left(\partial_{x} v\right)+2 b_{1}\left(\partial_{x} v\right)\right)$.

We can use the fifth equality of (A.4) to estimate $\left|b_{2}(v)\right|$ directly. Moreover, by virtue of the fourth equality of (A.5), the fourth equality of (A.4) and integration by parts, we observe that

$$
\begin{aligned}
b_{3}(v)+b_{2}\left(\partial_{x} v\right) & =\frac{1}{8} \int_{\Lambda}\left(-1+6 x^{2}-5 x^{4}\right) \partial_{x}^{5} v(x) d x+\frac{1}{2} \int_{\Lambda}\left(1-3 x^{2}\right) \partial_{x}^{4} v(x) d x \\
& =\frac{1}{2} \int_{\Lambda}(1-x)\left(1-2 x-5 x^{2}\right) \partial_{x}^{4} v(x) d x
\end{aligned}
$$

The same procedure, coupled with the last equality of (A.3), gives

$$
b_{3}(v)+b_{2}\left(\partial_{x} v\right)+2 b_{1}\left(\partial_{x} v\right)=\frac{1}{2} \int_{\Lambda}(1-x)\left(3-5 x^{2}\right) \partial_{x}^{4} v(x) d x .
$$

Consequently, $\left\|\partial_{x}^{4} v_{3,4, b}\right\|_{\chi^{(1,0)}} \leq c\left\|\partial_{x}^{4} v\right\|_{\chi^{(1,0)}}$. The previous statements imply (A.1) with $m=3, n=4$ and $r=6,5$, 4. Similarly, $\left\|\partial_{x}^{r} v_{4,3, b}\right\|_{\chi^{(-4+r,-3+r)}} \leq c\left\|\partial_{x}^{r} v\right\|_{\chi^{(-4+r,-3+r)}}$ for $m=4, n=3$ and $r=6,5,4$. 


\section{REFERENCES}

[1] Z. Belhachmi, C. Bernardi and A. Karageorghis, Spectral element discretization of the circular driven cavity, Part II: the bilaplacian equation. SIAM J. Numer. Anal., 38(2001), 1926-1960. MR1856237 (2002f:65173)

[2] C. Bernardi, M. Dauge and Y. Maday, Spectral Methods for Axisymmetric Domains, Series in Applied Mathematics, 3, edited by P. G. Ciarlet and P. L. Lions, Gauhtier-Villars \& North-Holland, Paris, 1999. MR.1693480(2000h:65002)

[3] C. Bernardi and Y. Maday, Spectral Methods, in Handbook of Numerical Analysis, Vol.5, Techniques of Scientific Computing, 209-486, edited by P. G. Ciarlet and J. L. Lions, Elsevier, Amsterdam, 1997. MR 1470226

[4] B. Bialecki and A. Karageorghis, A Legendre spectral Galerkin method for the biharmonic Dirichlet problem, SIAM J. Sci. Comput., 22(2000), 1549-1569. MR.1813286 (2001m:65178)

[5] J. P. Boyd, Chebyshev and Fourier Spectral Methods, Second Ed., Dover, Mineola, NY, 2001. MR.1874071 (2002k:65160)

[6] C. Canuto, M. Y. Hussaini, A. Quarteroni and T. A. Zang, Spectral Methods, Fundamentals in Single Domains, Springer, Berlin, 2006. MR2223552 (2007c:65001)

[7] O. Coulaud, D. Funaro and O. Kavian, Laguerre spectral approximation of elliptic problems in exterior domains, Comp. Meth. in Appl. Mech and Engr., 80 (1990), 451-458. MR 1067965

[8] E. H. Doha and A. H. Bhrawy, Efficient spectral-Galerkin algorithms for direct solution of fourth-order differential equations using Jacobi polynomials, Appl. Numer. Math., 58(2008), 1224-1244. MR2428974 (2009c:65330)

[9] W. N. Everitt, L. L. Littlejohn and R. Wellman, The Sobolev orthogonality and spectral analysis of the Laguerre polynomials $\left\{L_{n}^{-k}\right\}$ for positive integers $k^{*}$, J. Comp. Appl. Math., 171(2004), 199-234. MR2077205(2005i:42039)

[10] D. Funaro, Polynomial Approximations of Differential Equations, Springer-Verlag, Berlin, 1992. MR1176949 (94c:65078)

[11] D. Gottlieb and S. A. Orszag, Numerical Analysis of Spectral Methods: Theory and Applications, SIAM-CBMS, Philadelphia, 1977. MR.0520152 (58:24983)

[12] Guo Ben-yu, Spectral Methods and Their Applications, World Scientific, Singapore, 1998. MR:1641586 (2000b:65194)

[13] Guo Ben-yu, Gegenbauer approximation in certain Hilbert spaces and its applications to singular differential equations on the whole line, SIAM J. Numer. Anal., 37(2000), 621-645. MR 1740765 (2000k:65216)

[14] Guo Ben-yu, Jacobi approximations in certain Hilbert spaces and their applications to singular differential equations, J. Math. Anal. and Appl., 243(2000), 373-408. MR1741531 (2001b:65082)

[15] Guo Ben-yu and He Li-ping, The fully discrete Legendre spectral approximation of twodimensional unsteady incompressible fluid flow in stream function form, SIAM J. Numer. Anal., 35(1998), 146-176. MR.1618444(99f:76103)

[16] Guo Ben-yu and Jia Hong-li, Spectral method on quadrilaterals, Math. Comp., 79(2010), 2237-2264. MR2684363

[17] Guo Ben-yu and Shen Jie, Laguerre-Galerkin method for nonlinear partial differential equations on a semi-infinite interval, Numer. Math., 86(2000), 635-654. MR1794346(2001h:65152)

[18] Ben-yu Guo, Jie Shen and Li-lian Wang, Optimal spectral-Galerkin methods using generalized Jacobi polynomials, J. Sci. Comp., 27(2006), 305-322. MR2285783 (2008f:65233)

[19] Ben-yu Guo, Jie Shen and Li-lian Wang, Generalized Jacobi polynomials/Functions and their applications, Appl. Numer. Math., 59(2009), 1011-1028. MR2495135 (2010e:33013)

[20] Guo Ben-yu, Shen Jie and Xu Cheng-Long, Generalized Laguerre approximation and its applications to exterior problems, J. Comp. Math., 23(2005), 113-130. MR2118049|(2005m:65289)

[21] Guo Ben-yu and Wang Li-lian, Jacobi approximations and Jacobi-Gauss-type interpolations in non-uniformly Jacobi-weighted Sobolev spaces, J. Appr. Theo., 28(2004), 1-41. MR2063010 (2005h:41010)

[22] Guo Ben-yu, Wang Li-lian and Wang Zhong-qing, Generalized Laguerre interpolation and pseudospectral method for unbounded domains, SIAM J. Numer. Anal., 43(2006), 25672589. MR2206448(2007e:65129) 
[23] Ben-yu Guo and Tian-jun Wang, Composite generalized Laguerre-Legendre spectral method with domain decomposition and its application to Fokker-Planck equation in an finite channel, Math. Comp., 78(2009), 129-151. MR2448700 (2010g:65161)

[24] Guo Ben-yu and Wang Tian-jun, Composite Laguerre-Legendre spectral method for exterior problems, Adv. in Comp. Math., 32(2010), 2123-2142. MR2602220 (2011c:65274)

[25] Ben-Yu Guo and Tian-Jun Wang, Composite Laguerre-Legendre spectral method for fourthorder exterior problems, J. Sci. Comp., 44(2010), 255-285. MR2671289

[26] Guo Ben-yu and Xu Cheng-long, Mixed Laguerre-Legendre pseudospectral method for incompressible fluid flow in an infinite strip. Math. Comp., 72(2003), 95-125. MR2034112 (2004m:65157)

[27] Benyu Guo and Keji Zhang, On non-isotropic Jacobi pseudospectral method, J. of Comp. Math., 26(2008), 511-535. MR2431182(2009f:65270)

[28] Guo Ben-yu and Zhang Xiao-yong, A new generalized Laguerre spectral approximation and its applications, J. Comp. Appl. Math., 181(2005), 342-363. MR2146844 (2006e:65180)

[29] G. E. Karniadakis and S. J. Sherwin, Spectral/hp Element Methods for CFD, The Second Edition, Oxford Univ. Press, Oxford, 2005. MR2165335 (2006j:65001)

[30] Y. Maday, B. Pernaud-Thomas and H. Vandeven, Oneréhabilitation des méthods spèctrales de type Laguerre, Rech. Aérospat., 6(1985), 353-379. MR850680(88b:65135)

[31] G. Mastroianni and G. Monegato, Nystrom interpolants based on zeros of Laguerre polynomials for some Weiner-Hopf equations, IMA J. Numer. Anal., 17(1997), 621-642. MR1476342 $(98 \mathrm{j}: 45011)$

[32] G. Mastroianni and D. Occorsio, Lagrange interpolation at Laguerre zeros in some weighted uniform spaces, Acta Math. Hungar, 91(2001), 27-52. MR.1912359 (2003k:41005)

[33] Shen Jie, Stable and efficient spectral methods in unbounded domains using Laguerre functions, SIAM J. Numer. Anal., 38(2000), 1113-1133. MR1786133 (2001g:65165)

[34] G. Szegö, Orthogonal Polynomials, Amer. Math. Soc., Providence, RI, 1959.

[35] Wang Xing-hua, On Hermite interpolation (in Chinese), Science in China, Ser. A: Mathematics, 37(2007), 945-954. MR2390478(2009c:41011)

[36] Xu Cheng-long and Guo Ben-yu, Laguerre pseudospectral method for nonlinear partial differential equation, J. Comp. Math., 20(2002), 413-428. MR1914675 (2003e:65184)

Department of Mathematics, Shanghai Normal University, Shanghai, 200234, China - And - Scientific Computing Key Laboratory of Shanghai Universities, Division of Computational Science of E-institute of Shanghai Universities

E-mail address: byguo@shnu.edu.cn

Department of Applied Mathematics, Shanghai Finance University, Shanghai, 201209, CHINA

E-mail address: taosun80@yeah.net

Department of Mathematics, Xuzhou Normal University, Xuzhou, 221116, China

E-mail address: zcxz1977@163.com 